\title{
Dynamic interaction of BRCA2 with telomeric G- quadruplexes underlies telomere replication homeostasis
}

\author{
Junyeop Lee
}

Seoul National University

Keewon Sung

Seoul National University

So Young Joo

Seoul National University

Jun-Hyeon Jeong

Seoul National University

Seong Keun Kim

Seoul National University

Hyunsook Lee ( $\nabla$ HL212@snu.ac.kr)

Seoul National University https://orcid.org/0000-0002-2251-5019

Article

Keywords: BRCA2, G-quadruplex, Telomere, MRE11, Telomere Replication

Posted Date: September 10th, 2021

DOl: https://doi.org/10.21203/rs.3.rs-754735/v1

License: (9) (i) This work is licensed under a Creative Commons Attribution 4.0 International License. Read Full License

Version of Record: A version of this preprint was published at Nature Communications on June 13th, 2022. See the published version at https://doi.org/10.1038/s41467-022-31156-z. 


\title{
Dynamic interaction of BRCA2 with telomeric G-quadruplexes underlies telomere replication homeostasis
}

\author{
Junyeop Lee ${ }^{1 \S}$, Keewon Sung ${ }^{2 \S}$, So Young Joo ${ }^{1}$, Jun-Hyeon Jeong ${ }^{1}$, Seong Keun Kim ${ }^{2 *}$, and \\ Hyunsook Lee ${ }^{1^{*}}$ \\ ${ }^{1}$ Department of Biological Sciences \& IMBG, Seoul National University. \\ Seoul 08826, South Korea \\ ${ }^{2}$ Department of Chemistry, Seoul National University. \\ Seoul 08826, South Korea
}

$\S$ These authors contributed equally

*Correspondence:

1. H.L., Department of Biological Sciences \& IMBG, Seoul National University. 1 Gwanak-Ro, Gwanak-Gu, Seoul 08826, South Korea

Email: $\underline{\text { HL212@snu.ac.kr }}$

2. S.K.K., Department of Chemistry, Seoul National University. 1 Gwanak-Ro, Gwanak-Gu, Seoul 08826, South Korea

Email: seongkim@snu.ac.kr

Running title: Mode of Interaction of BRCA2 with the telomere G-quadruplex

Keywords: BRCA2; G-quadruplex; Telomere; MRE11; Telomere Replication 


\begin{abstract}
BRCA2-deficient cells undergo telomere shortening upon collapse of stalled replication forks, particularly during lagging-strand telomere synthesis. The molecular mechanism underlying fork collapse remains unclear. Here we find that the BRCA2 C-terminus, which includes an OB-fold, specifically interacts with G-quadruplex (G4) structures generated during lagging-strand telomere replication. We demonstrate that BRCA2 associates with Gtriplex (G3)-derived intermediates using electrophoretic mobility shift assay and singlemolecule FRET. These G3 intermediates form during direct interconversion between parallel and non-parallel G4 structures. Intriguingly, MRE11 nuclease can resect G4-forming telomere sequences, a function that is inhibited by BRCA2. BRCA2 depletion consistently resulted in increased telomeric damage, which was relieved by MRE11 knockdown. These data suggest that BRCA2 interaction with telomeric G4 prevents MRE11-mediated resection. The specific interaction between BRCA2 and G4 therefore contributes to telomere stability and genome integrity.
\end{abstract}




\section{Introduction}

Eukaryotic chromosomes are linear leading to the 'end replication problem' where telomeres shorten with each replication cycle. This effectively means that the protective effect of telomeres is lost over time, resulting in decreased genome integrity. Differentiated cells usually lack telomerase activity, and thus face senescence due to progressive telomere shortening. Overcoming cellular senescence is a critical step during cell transformation in tumorigenesis: the majority of cancer cells have active telomerase. Approximately $\sim 10-15 \%$ of cancers exhibit ALT (Alternative Lengthening of Telomeres), a mechanism that maintains and elongates telomeres allowing cancer cells to thrive and proliferate.

Telomere repeat array sequences are well conserved from yeast $\left.\left(\mathrm{TG}_{2-3(\mathrm{TG}}\right)_{1-6}\right)$ to human $(\text { TTAGGG })^{1,2}$. Notably, consecutive guanine repeats can interact through non-canonical Hoogsteen base-pair formation, where the guanine becomes both the donor and the acceptor of the hydrogen, instead of engaging in Watson-Crick pairing ${ }^{3}$. As a result, Hoogsteen pairs of four guanines can form a flat plane called a G-tetrad ${ }^{4}$. Two or more G-tetrads can stack to form a chair or basket-like structure called a G-quadruplex (G4) . Intriguingly, singlestranded G-rich telomeric DNA is also able to form G4 structures in a physiological environment ${ }^{6,7}$. During replication of the lagging strand at telomeres, the single-stranded telomeric repeat can potentially fold into G4, thus becoming an obstacle for replication fork progression. Left unresolved, G4 can cause telomere erosion and result in aberrant recombination $^{8-10}$. At the same time, folding into G4 can control access to telomerase, thereby regulating telomere maintenance ${ }^{11,12}$. This may explain why G-rich telomere repeat has been conserved during eukaryotic evolution. 
Germ-line mutation of the breast cancer susceptibility gene, $B R C A 2$ predisposes carriers to cancers of the breast, pancreas, ovary, and other tissues ${ }^{13,14}$. We and others have shown that disruption of $B R C A 2$ results in telomere shortening ${ }^{15,16}$ due to the failure to protect replication fork collapse on the lagging-strand ${ }^{16}$. MRE11 functions in DNA resection of the stalled replication forks, and it is possible that BRCA2 protects telomeres from this potentially damaging resection activity. Here, we examined the mechanism by which BRCA2 maintains telomere replication homeostasis. Using molecular and biophysical methods, we show that BRCA2 directly binds to an intermediate DNA secondary structure, G3 or G3:G4, which forms during interconversion between parallel and non-parallel G4 without the need to pass through an unfolded single-stranded DNA intermediate. Furthermore, MRE11 nuclease recognizes and resects telomere G4, and the binding of BRCA2 to G3-derived intermediates protects telomeres from resection by MRE11. Our data indicate how BRCA2 protects telomeres and prevents their loss to thereby promote genome integrity.

\section{Results}

\section{BRCA2 binds to telomeric G-quadruplex structures}

We previously observed that BRCA2 localizes to $\sim 10 \%$ of telomeres during S phase ${ }^{16}$. We used immunoFISH to confirm that in GFP-tagged BRCA2 localizes to telomeres in HeLa cells (NFLAP-HeLa ${ }^{17,18}$ ) as well (Supplementary Fig. S1). Absence of BRCA2 results in fragile telomeres, which led us to speculate that BRCA2 may be critical in telomere maintenance through the protection of stalled replication forks ${ }^{16}$. To unveil the mechanistic role that BRCA2 plays in telomere replication, we investigated the molecular nature of BRCA2 binding to S phase telomeres. 
BRCA2 is a $\sim 420 \mathrm{kDa}$ protein with no apparent homology to other known proteins.

However, there are three oligonucleotide/oligosaccharide-binding folds (OB-folds) at the Cterminus, which show limited homology to the OB-fold of RPA, which is known to bind to single-stranded DNA ${ }^{19}$. Notably, POT1, a member of the telomeric Shelterin protein, specifically binds to single-stranded telomeric DNA and also possesses OB-folds ${ }^{20-22}$. The BRCA2 DNA-binding domain (DBD), which contains the OB-folds, binds to single-stranded DNA in complex with the cofactor DSS1 (Deleted in split-hand/split-foot syndrome protein). This complex coordinates homologous recombination (HR) during double-stranded DNA break (DSB) repair. The structure of the DBD in complex with DSS1 and a random ssDNA has been solved ${ }^{19}$ (DBD, Fig. 1a).

Considering this information, we asked whether the DBD of BRCA2 has telomere binding activity in the absence of DSS1. In order to test this, three GST-tagged proteins, encompassing the DBD at the C-terminus of human BRCA2 (B2-7, B2-8, B2-9) ${ }^{23}$, were purified. Binding of these recombinant proteins to radio-labeled G-rich single-strand or Crich single-strand oligonucleotides representing telomeric sequences was tested using EMSA (Electrophoretic mobility shift assay). While none of the recombinant fragments associated with the C-rich strand, the OB-fold-containing species (B2-8) specifically bound to the Grich telomere sequence (Supplementary Fig. S2).

To further extend the study, MBP (maltose-binding protein)-tagged BRCA2OB (a.a. 26703185), which contains the OB-fold, was purified from E. coli (Fig. 1a) and subjected to EMSA. Single-stranded G-rich telomere arrays (TelG5, (GGTTAG)5), single-stranded C-rich arrays (TelC5; (CTAACC)5), or double stranded oligonucleotides (TelG5:TelC5) were endlabeled with $\gamma-{ }^{32} \mathrm{P}-[\mathrm{ATP}]$ then employed as probes in EMSA (Fig. 1b \& Supplementary Table 1). The OB-fold of BRCA2 specifically bound to single-stranded G-rich telomere array 
(TelG5), but not to single-stranded C-rich (TelC5) nor to double-stranded telomere repeats (Fig. 1b; Supplementary Table 1). The binding of BRCA2OB to TelG5 increased in a dosedependent manner (Fig. 1c, lanes 1-4). Furthermore, adding unlabeled cold TelG5 abolished formation of the BRCA2OB-TelG5 complex (Fig. 1c, lane 5), confirming that the binding of BRCA2OB to TelG5 is specific.

In TelG5, there are four consecutive TTAGGG repeat arrays with GG and TTAG flanking sequences at the 5' and 3' end, respectively (Supplementary Table 1). Therefore, TelG5 is capable of forming G4 structures. We asked whether BRCA2OB can interact with this telomeric G4. For this, increasing number of telomere repeat arrays were tested for binding to BRCA2OB. BRCA2OB binds to single-stranded G-rich oligonucleotides with more than 4 repeats but not to those with less (Fig. 1d): BRCA2OB binding was apparent in TelG5 and TelG6, which can fold into G4 but BRCA2OB did not associate with TelG3 or TelG4, which can fold into G-tetrads but cannot stack to form G4 (Fig. 1d).

It should be emphasized that BRCA2OB associates with TelG5 in the absence of DSS1. This suggests that the nature of BRCA2 binding to G-rich telomeres is distinct from non-sequence specific BRCA2-DSS1 interaction with single-stranded DNA during $\mathrm{HR}^{19,24}$. To test this idea, we utilized a mutant construct termed TelG5-GAG (TTAGAG repeat), where GGG in TelG5 is replaced with GAG to disrupt folding into G4 structures. Circular dichroism (CD) spectra confirmed that TelG5-GAG is mainly composed of unfolded single-stranded DNA (Supplementary Fig. S3). By comparing the interaction of BRCA2OB with TelG5 and TelG5GAG, we confirmed that BRCA2OB associates with G4 telomeres, but not singlestranded DNA (Fig. 1e). 
Next, we examined the effect of the G4-stabilizing ligand pyridostatin (PDS) ${ }^{25,26}$ on the BRCA2OB-TelG5 interaction. PDS is a potential anti-cancer drug because it induces DNA damage $^{27-29}$. PDS-bound G4 telomeres would likely remain unresolved, and then undergo resection by G4-specific nucleases, which would be lethal to cancer cells. When PDS was pre-incubated with TelG5, it inhibited the BRCA2OB-TelG5 interaction in a dose-dependent manner (Fig. 1f). Together, these results suggest that BRCA2OB specifically interacts with G4 at telomeres.

\section{Unique mode of BRCA2 interaction with telomeric G4}

Sequences that can form G4 are found throughout the genome, especially at promoters ${ }^{30-34}$, including the $C-M Y C$ promoter $^{35}$. The likelihood that BRCA2 is involved in transcriptional control is low, in contrast to BRCA1. We asked if BRCA2OB binding discriminates telomere G4 from non-telomeric G4. To test this, we compared BRCA2OB binding to telomeric G4 sequence vs. the C-MYC promoter or an artificially designed oligonucleotide (Supplementary Table 1) using EMSA. We found that BRCA2OB specifically associated with telomere G4; it bound markedly less to $C-M Y C$ promoter DNA or the artificially designed G4, while interaction with telomere G4 was significant (Supplementary Fig. S4).

Although mammalian telomere is mostly composed of TTAGGG repeat arrays, variations in the sequence exist at the proximal or subtelomeric regions as well as within some telomeric regions. These variants are referred to as telomere variant repeats (TVRs): TCAGGG, TGAGGG, CTAGGG, TTGGGG, and TTTGGG ${ }^{36-40}$. Notably, variations in TVRs are restricted to the non-guanine loop, leaving intact the consecutive guanine repeats that are 
responsible for stacking square-planar tetrads. This implies that the ability to fold into G4 may be a critical feature that is characteristic of telomeres.

In EMSA, BRCA2OB binding was observed to TelG (typical TTAGGG), TelG-TCA (TCAGGG), and TelG-TGA (TGAGGG) variants. In comparison, binding of BRCA2OB to TelG-CTA (CTAGGG) and the unfolded mutant control TelG-GAG was not detected (Supplementary Fig. S5 and Supplementary Table 1).

In order to assess the binding affinity of BRCA2OB for G4-forming telomere, we performed EMSA and measured the dissociation constant $\left(K_{d}\right)$ of BRCA2OB for three different telomeric G4 sequences (Fig. 2a). BRCA2OB had a $K_{d}=4.4 \mu \mathrm{M}$ of for TelG5, $K_{d}=456$ $\mathrm{nM}$ to TelG5TTT, and $K_{d}=1.4 \mu \mathrm{M}$ to TelG5T2G4 (Fig 2a). Notably, the $K_{d}$ of POT1 binding to G-rich ssDNA representing telomeres is $38.2 \mathrm{nM}^{41}$; TLS/FUS to telomeric $\mathrm{G} 4$ is $37 \mathrm{nM}^{42}$; and nucleolin to telomeric $\mathrm{G} 4$ is $2.574 \mu \mathrm{M}^{43}$.

Notably, BRCA2OB binding increased $\sim 13$ times and $\sim 5$ times more in assays using TelG5TTT (TTTGGG repeats) and TelG5T2G4 (TTGGGG repeats). These sequences represent the telomeres of ciliates ${ }^{44}$ and Tetrahymena ${ }^{45,46}$, respectively, and are also found in cancers $^{47}$ (Fig. 2b). Next we compared the binding of the OB folds from the Shelterin complex protein POT1 (POT1OB) to BRCA2OB. Notably, POT1 binding has been reported to disrupt the G4 structure ${ }^{48}$. The binding mode of POT1 to telomere variants contrasted with that of BRCA2OB: POT1OB bound to TelG5 (TTAGGG repeats) strongly as expected, but it did not bind to the other variants BRCA2OB interacts with (Fig. 2b). These data are consistent with the notion that BRCA2 binds to telomeres during S phase when POT1 binding must be released for replication. Structural information may reveal the basis of this differential mode of interaction in the future. 
Next, we asked whether full length BRCA2 also binds telomere variants. Due to the large size ( $420 \mathrm{KDa})$, purification of recombinant BRCA2 is not feasible. Therefore, we utilized NFLAP-BRCA2-HeLa cells, which stably express EGFP-tagged BRCA2 in a BAC (bacterial artificial chromosome) clone ${ }^{17}$. EGFP-tagged full length BRCA2 was immunoprecipitated with an anti-GFP antibody from the cell lysate. The immunoprecipitate was incubated with ${ }^{32} \mathrm{P}$-labeled probes, followed by several washes and subsequent dot blotting on positively charged nylon membrane (Fig. 2c). Consistent with BRCA2OB, full length BRCA2 exhibited the strongest binding to TelG5TTT, followed by TelG5T2G4, then TelG5 (Fig. 2d). Full length BRCA2 therefore has similar binding properties to recombinant BRCA2OB.

\section{Direct observation of G4 dynamics and BRCA2 interaction.}

Telomere G4 can form various topologies including parallel, anti-parallel, and hybrid conformations $^{49-53}$, depending on the strand polarities, the number of guanine repeats, the length and sequence of the non-guanine loop, the concentration and type of monovalent metal cations, and molecular crowdedness ${ }^{54-58}$. In addition, single-molecule analysis has identified intrinsic structural rearrangements between three detectable conformations (i.e., the parallel (P), non-parallel (NP), and unfolded (UF)), indicating the dynamic nature of $\mathrm{G} 4^{54,59,60}$.

To reveal the molecular characteristics of the interaction between BRCA2 and telomeric G4 in detail, we utilized single-molecule FRET (smFRET) spectroscopy. A partially duplexed single-stranded oligonucleotide of TelG5, was labeled with the FRET donor (Cy3) and acceptor (Cy5) at opposite ends (Fig. 3a, top, and Supplementary Table 2). This 
oligonucleotide was immobilized on a quartz surface for direct observation of conformational dynamic shifts between UF, NP, and P conformations (Fig. 3a).

In circular dichroism (CD) spectra, TelG5 exhibited two positive peaks under $\mathrm{K}^{+}$-rich conditions: a major peak at $\sim 290 \mathrm{~nm}$ and a minor peak at $\sim 265 \mathrm{~nm}$ (Fig. 3b). According to previous reports, the $290 \mathrm{~nm}$ and the $265 \mathrm{~nm}$ peaks in CD spectra correspond to the characteristics of NP and P folding, respectively ${ }^{52}$. In the smFRET histogram, TelG5 exhibited two peaks in the presence of $100 \mathrm{mM} \mathrm{KCl}$ : a major middle-FRET state $(E \sim 0.47)$ and a minor high-FRET state $(E \sim 0.68)$ (Fig. 3c). Correlating with the CD spectra, we assigned the major middle-FRET state as NP and the minor high-FRET state as the $\mathrm{P}$ conformation (Fig. 3c \& d). This agrees with previous studies that analyze G4 constructs ${ }^{61-}$ 63. A small fraction of the low-FRET state $(E \sim 0.23)$ was assigned as unfolded (UF), because it was identical to the smFRET value of TelG5GAG (Supplementary Fig. S6). $\quad 48 \%$ of individual single-molecule time trajectories using TelG5 showed frequent transitions among the UF, NP, and $\mathrm{P}$ conformations in $100 \mathrm{mM} \mathrm{KCl}$ buffer, confirming that telomeric G4 is highly dynamic and heterogeneous in nature (Fig. 3d).

To directly monitor the BRCA2-telomere G4 interaction, we added $1 \mu \mathrm{M}$ of recombinant BRCA2OB to the surface-immobilized TelG5 DNA. As a result, a new FRET peak appeared (E 0.57) between the NP and P conformation peaks (Fig. 3e). This peak increased with increasing concentration of BRCA2OB (Supplementary Fig. S7). The new FRET state was consistently identified in single-molecule time trajectories upon addition of BRCA2OB, and was observed right after the FRET transitions between the NP and P conformations (Fig. 3f). Taken together, the new FRET state arises from a BRCA2OB-bound (BD) conformation (Fig. 3f, BD state). These results corroborate the interaction between BRCA2 and the dynamic telomere G4 at the single-molecule level. The data also suggest that 
BRCA2OB may recognize one of the telomeric G4: P, NP conformations and other potentially short-lived structures.

\section{Biophysical properties of telomeric G4 dictates BRCA2 binding}

To further analyze the characteristics of the interaction between BRCA2OB and the G4 telomere, we compared the biophysical properties of TelG5 with the three previously characterized G4 variants (TTA to TAA for TelG5TAA; TTT for TelG5TTT; TT for TelG5TT (Supplementary Table 2)) using smFRET spectroscopy ${ }^{54}$. The relative fraction of the $\mathrm{P}$ conformation in smFRET markedly increased in TelG5TTT and TelG5TT, compared to TelG5 and TelG5TAA, as reported ${ }^{54}$. The UF (unfolded) fraction was highest in smFRET data from TelG5TAA (Fig. 4a). Similar results were obtained from the CD spectra (Supplementary Fig. S8a).

Upon addition of BRCA2OB to these variants, a drastic difference in binding properties was measured in smFRET. The BD fraction was $\sim 7 \%$ and $\sim 8 \%$ for TelG5TAA and TelG5, respectively, in the presence of $100 \mathrm{nM}$ of BRCA2OB (Fig. 4b, left). In the presence of 1 $\mu \mathrm{M}$ of BRCA2OB, the BD fraction remained at $\sim 20 \%$ for both TelG5TAA and TelG5 (Supplementary Fig. S8b). However, the BD fraction of TelG5TTT and TelG5TT significantly increased to $\sim 60 \%$ after adding $100 \mathrm{nM}$ BRCA2OB (Fig. $4 \mathrm{~b}$, right), and became saturated in the presence of $1 \mu \mathrm{M}$ of BRCA2OB (Supplementary Fig. S8b). In EMSA, it was apparent that TelG5TTT and TelG5TT retained the strongest binding to BRCA2OB (Supplementary Fig. S9). These results led us to investigate the dynamic nature of telomeric G4 structures in association with differential BRCA2OB-binding properties. 
First, the individual time trajectories of FRET transitions were analyzed by calculating the dwell time in each conformation (Fig. 4c \& Supplementary Fig. S10). The average dwell time in the UF conformation (Fig. 4c, gray) was the highest and four-times longer in TelG5TAA (4.3 s), and this showed the weakest interaction with BRCA2OB, further supporting that BRCA2OB binds inefficiently to UF-ssDNA. The dwell time in the NPconformation remained constant for all telomere variants (Fig. 4c, green). Meanwhile, the dwell time in the P conformation (Fig. 4c, red) was nearly twice as long in TelG5TTT and TelG5TT (5.2 s and 4.3 s respectively; Supplementary Fig. S10). However, BRCA2OB binding to TelG5TTT and TelG5TT was 10-fold higher in EMSA (Fig. 4f \& Supplementary Fig. S9). This marked difference cannot be explained by the 2 -fold increment in the Pconformational dwell time in TelG5TTT or TelG5TT (Fig. 4c).

To understand these G4 dynamics further, the transition density between the three interchangeable UF, NP, and P conformations was examined (Fig. 4d). Interestingly, the TelG5TAA and TelG5 substrates mainly displayed transitions between the UF and NP and between the UF and P conformations (Fig. 3d, 4d and Supplementary Fig. S11a). This indicates that the NP and P conformations structurally rearrange through complete unfolding and refolding in TelG5. In stark contrast, TelG5TTT and TelG5TT predominantly underwent direct transitions between the NP and P conformations (Fig. 4d, e \& Supplementary Fig. S11b), indicating that the NP-P conformational rearrangement occurs through partially folded intermediate structures without passing through the UF state.

Next, the density of the NP-P direct transition was quantified by measuring the relative fraction of both $\mathrm{NP} \rightarrow \mathrm{P}$ and $\mathrm{P} \rightarrow \mathrm{NP}$ transitions multiplied by the fraction of molecules undergoing conformational transitions (Supplementary Fig. S12). Notably, the density of the NP-P direct transition matched the relative binding affinity of BRCA2OB to G4 
substrates (Fig. 4f). This was true even in the case of TelG5T2G4, in which G4 conformations are more promiscuous due to the formation of four stacks of G-tetrads (Supplementary Fig. S13). Taken together, these results imply that BRCA2OB selectively interacts with an intermediate topology formed during the NP-P conformational transition in telomeric G4 (Fig. 4g).

\section{BRCA2 recognizes and binds to G-triplex-derived intermediates}

Previous studies using molecular dynamics simulations ${ }^{64-66}$, stopped-flow kinetic measurement ${ }^{67,68}$, NMR spectroscopy ${ }^{69,70}$, and single-molecule experiments ${ }^{71-73}$ have proposed the formation of intermediate structures as part of the G4-folding and unfolding pathway. These intermediates include the G-triplex (G3) and G-hairpin (G2). Taking this information into account, we asked whether BRCA2OB interacts with these intermediates during interconversion between the NP-P conformations (Fig. 4d). To test this idea, we examined the conformational dynamics of a construct, termed TelG5-G3, where the third GGG triplet within the TelG5 sequence was changed to GTG, resulting in three intact GGG triplets with a long loop of TTAGTGTTA (Supplementary Table 2). The effect of BRCA2OB addition was then tested (Fig. 5a).

In smFRET, the majority of TelG5-G3 alone resided in the low-FRET UF conformation. Notably, a considerable fraction populated at the two higher-FRET folded states (Fig. 5b, upper). The FRET efficiency of the middle-FRET state $(E \sim 0.57)$ was similar to that of G4 conformations (i.e. P and NP). Therefore, this middle-FRET peak is likely to consist of the G3 with the long loop (Fig. 5a) and another type of structure consisted of a mixture of Gtriads and G-tetrads that may fold through interactions between the $\mathrm{G} 3$ and the remaining $\mathrm{G}$ 
bases in the long loop sequence (termed as loop-associated G3:G4) $)^{74,75}$. Likewise, the highFRET state $(E \sim 0.83)$ was designated as representing tail-associated G3:G4, an interaction between the G3 and extra $\mathrm{G}$ bases in the tail sequence. These structural characterizations were supported by additional experiments with TelG4 [(GGTTAG) 4 ] and TelG4-G3 [(TTTTAG)-(GGTTAG)2-(GGTTAT)] (Supplementary Fig. S14). Interestingly, previous reports have suggested that G3:G4 is an intermediate during G4 conformational transitions $\mathbf{s}^{74,75}$.

Notably, BRCA2OB addition resulted in a rapid growth of the middle-FRET population (G3 and loop-associated G3:G4) and a slight increase in the high-FRET (tail-associated G3:G4) fraction for TelG5-G3, without perturbing their FRET values (Fig. 5b, lower). This result indicates that BRCA2OB directly recognizes the G3 and G3:G4 structures. A similar increase in the middle-FRET fraction was observed in TelG5TTT-G3 (Fig. 5c), where the third GGG triplet within the TelG5TTT variant is replaced with GTG (Supplementary Table 2). Note that the FRET efficiency of the middle-FRET state for TelG5-G3 and TelG5TTTG3 perfectly matches that of the BRCA2OB-bound (BD) state $(E \sim 0.57)$ when the TelG5 and TelG5TTT sequences are used (Fig. 5b, shaded, adopted from Fig. 3e \& Supplementary Fig. S8b).

The binding of BRCA2OB to the G-triplex-derived structures (i.e., G3 and G3:G4) was confirmed by EMSA: compared to TelG5, BRCA2OB binding was increased by three-fold for TelG5-G3 and 10-fold for TelG5TTT (Fig. 5e). These results suggest that BRCA2 associates with the G4 substrates primarily through selective interaction with the G-triplexderived intermediates, which form during the NP-P conformational interconversions (Fig. 5f, box with broken line). The NP-P conversion via UF-ssDNA (Fig. 5g, lower, unboxed) may also involve the G3-related intermediates. However, BRCA2 binds poorly to G4 substrates 
that exhibit frequent UF-mediated conformational transitions (Fig. 4d and f); i.e.,the half-life of the G3 intermediates in this process, if they exist, is markedly shorter than G3 intermediates formed during the direct NP-P transition. Taken together, BRCA2 recognition and binding to G3 intermediates may reduce folding into G4 (Fig. 5f).

\section{MRE11 as the nuclease that attacks G4 structures in telomeres.}

As processivity and thus replication is slower during lagging strand synthesis, we hypothesized that a secondary structure, such as G4, could form from the exposed guaninerich single strand during telomere lagging strand synthesis. Unresolved G4 can become an obstacle for the progression of the replication fork ${ }^{16}$. The above results indicate a dynamic interaction between BRCA2 and the telomeric G4. In addition it is known that MRE11 resects stalled replication forks ${ }^{76,77}$. Therefore, it is reasonable to think that there is interplay between BRCA2, telomeric G4, and MRE11. However, MRE11 might also be the nuclease that can recognize and resect the telomeric G4. Interestingly, an older report suggested that yeast Mre11 alone, or as part of a sub-complex, can resect the 5'-end of G-rich single-stranded DNA, the G-tetrad, or the center of TGTG repeats in duplexed DNA ${ }^{78}$. These results suggest that MRE11 may be involved in telomeric G4 digestion.

To test whether MRE11 attacks telomeric G4, we purified the nuclease-active form of MRE11 (MRE11N) from E. coli ${ }^{79,80}$ (Fig. 6a) and conducted a nuclease assay with the telomere variants. The endo- and exo-nucleolytic activities of recombinant MRE11N was confirmed by its ability to cleave the hairpin-like secondary structured DNA, DAR134, by comparing it with the nuclease-dead mutant MRE11N-H129N ${ }^{79}$ (Supplementary Fig. S15). Consistently, TelG5GAG, the unstructured single strand, was completely degraded upon 
incubation with MRE11N. This result confirms that recombinant MRE11N retains its exonuclease activity (Supplementary Fig. S15).

Upon addition of MRE11N, telomere G4 variants with distinct topological distributions reacted differently (Fig. 6b-e). MRE11 efficiently digested TelG5 in a dose-dependent manner (Fig. 6c, lane 2-3). MRE11 also showed efficient activity toward the G3 folding construct TelG5-G3, but with less efficiency (Fig. 6b, lane 2-3). Note that TelG5-G3 exhibit UF-ssDNA, which can be the substrate of MRE11 through its exonuclease activity (Fig. 5b). Intriguingly, TelG5TTT was most vulnerable to MRE11N in that almost all of the substrate was degraded (Fig. 6d, lane 2-3). To assess whether MRE11 can resect telomeric G4 in a structure-dependent manner, we utilized TelG3GGG, where the TelG5 overhangs were removed leading to reduced dynamicity ${ }^{81}$. Interestingly, MRE11N also exhibited nuclease activity on TelG3GGG (Fig. 6e, lane 2-3), corroborating that MRE11 can recognize the telomeric G4 and resect this structure.

Next, we asked whether the addition of BRCA2OB affected MRE11 nuclease activity towards telomere-specific G4. In order to test this, purified BRCA2OB was pre-incubated with the probes in reaction buffer before adding MRE11N. BRCA2 incubation protected telomeric G4 from MRE11-mediated resection. TelG3GGG was fully protected by BRCA2OB from MRE11 (Fig. 6e, lane 4-5), and $5 \mu \mathrm{M}$ of BRCA2OB was enough to prevent resection of TelG5 (Fig. 6c, lane 4-5). TelG5TTT was moderately protected by BRCA2OB in a dose-dependent manner (Fig. 6d, lane 4-5). BRCA2OB preincubation did not fully protect the MRE11-mediated degradation in TelG5-G3 that there were smears representing the degradation (Fig.6b, lane 5). Smears may be the result of the combination of BRCA2OBbound G3 and the products of MRE11's exonuclease activity towards UF-ssDNA. Taken together, these results imply that MRE11 specifically targets telomere G4. In addition 
BRCA2 binding to the intermediate G3 or G3:G4 conformations (Fig. 5) prevents MRE11mediated digestion.

\section{BRCA2 protects telomeric G4 from MRE11-mediated resection during telomere replication}

Finally, we asked if the interplay between BRCA2, telomere G4, and MRE11 is observed during telomere replication in vivo. In order to test this, we took advantage of conditional Brca2-deficient mouse embryonic fibroblasts (MEFs), that is immortalized by SV40 LT. In this immortalized mouse fibroblast, which is Telomerase-negative and Brca2-depletioninducible (TBI), Brca2 can be depleted by the activation of Cre recombinase through the treatment with tamoxifen (4-OHT) ${ }^{82}$. Note that Brca2 depletion is linked to induction of alternative lengthening of telomeres (ALT) as these cells lack telomerase ${ }^{82}$.

The TBI fibroblasts were treated with 4-OHT to deplete Brca2 (Fig. 7a, time 0). Twentyfour hours later, cells were transduced with lentiviral-shMrel1 or control (lentiviral-shGFP). Forty-eight hours after shRNA introduction, TBI fibroblasts were serum starved for 16 hours to synchronize the cells in G1/S boundary. Cells were then released into S phase for $4 \mathrm{~h}$ with the addition of $16 \%$ serum. At this stage, cells were treated with the G4 stabilizer PDS or left untreated. Finally, cells were fixed and subjected to immunostaining, using antiTRF1 and anti- $\gamma-\mathrm{H} 2 \mathrm{AX}$ antibodies to assess telomeric damage (Fig. 7a).

When Brca2 was intact, PDS treatment or the depletion of Mre11 barely affected telomere integrity, as the number of $\gamma$-H2AX-positive telomeres per cell was low (Fig. 7b \& c, 4OHT). In contrast, Brca2 depletion increased telomere damage by two-fold (Fig. $7 \mathrm{~b} \& \mathrm{c}$, 
+4-OHT). Telomere damage in Brca2-depleted cells was further increased to four-fold by PDS treatment (Fig. 7b \& c, +4-OHT; +PDS). However, when Mre11 was depleted (Fig. 7d), telomere damage due to the depletion of Brca2 was reduced (Fig. 7c, +4-OHT; +shMre11). Mre11 depletion also reduced PDS-induced telomere damage in Brca2depleted cells to levels that are comparable to Brca2 abrogation alone (Fig. 7c, +4-OHT; +PDS; +shMre11). Similar results were obtained with the treatment of Mirin ${ }^{83}$, an MRE11 inhibitor (Supplementary Fig. S16). These results confirm that BRCA2 prevents MRE11mediated collapse of G4-stalled replication forks.

\section{Discussion}

In this study, we provide compelling evidence that BRCA2 interacts with dynamic telomeric G4 through selective binding to G3-derived intermediates, which form during interconversion between parallel and non-parallel telomeric G4 conformations (Fig. 7e, upper). Furthermore, we identified MRE11 as the nuclease that attacks telomeric G4. Association of BRCA2 with G3 reduces G4, which can be targeted by MRE11. Therefore, interaction of BRCA2 with dynamic G4 structures at telomeres results in protection of telomeres from MRE11mediated resection during S phase (Fig. 7e, lower). These results lead to the interesting hypothesis that BRCA2-bound telomeres, G3 or G3:G4, may be resolved by a specific helicase. In this way, BRCA2 association with telomeres guarantees telomeric homeostasis during replication. However, there may be other nucleases which can resect G4. How the nucleases work together, and identification of the G3-relieving helicase, warrant future investigation (Fig. 7e, lower). 
We speculate that BRCA2 interaction with telomeric G4 evolved to maintain genome integrity. It is noteworthy that guanine-rich telomere repeats have been conserved over 1.3 billion years of eukaryotic evolution. Conservation of guanine-richness at telomeres, and folding into G4 structures, may inhibit telomeric access by the DNA recombination machinery or telomerase. Consistent with this hypothesis, telomeric G4 is known to inhibit DNA damage regulators from acting at telomeres ${ }^{61,84,85}$. Structural dynamics of telomeric G4 also affects the accessibility of RAD51, WRN, and BLM to telomeres ${ }^{81}$. Binding of POT1/TPP1 and RPA to the telomere also depends on the topology of the G4 conformation ${ }^{61}$.

However, G4 serves as an obstacle to replication as well. Particularly during lagging-strand telomere synthesis, relatively slow synthesis of Okazaki fragments leaves the G-rich telomere template exposed, resulting in folding into G4. Our results unravel the long-standing question of how specific telomere sequences are linked to telomere function and contribute to genome integrity. BRCA2 binds to G3-derived intermediates during interaction with telomeric G4, while MRE11 recognizes and attacks these sequences. These results provide direct evidence that G4 dynamics can be exploited for specific molecular recognition. We propose that the biophysical nature of dynamic G4 controls accessibility: telomere G4 dynamics may function as a gatekeeper that blocks unwanted interactions, but allows access to factors that are required for telomere maintenance during replication.

Although further structural characterization is required in order to uncover a detailed mechanism, our in vitro data show that MRE11 exhibits strong nuclease activity towards the G4 substrates. The absence of BRCA2 results in telomere damage in cells, this is further exacerbated upon G4 stabilizer PDS treatment. Depletion of MRE11 alleviated the telomere damage induced by G4. Altogether, these results strongly speak to the fact that the dynamic 
interplay between BRCA2, telomere G4, and MRE11 guarantees telomere replication homeostasis.

Recently, we showed that Brca2 deficiency results in induction of alternative lengthening of telomeres (ALT) through the instigation of the break-induced replication (BIR) ${ }^{82,86}$. According to the current study, it is possible that the absence of BRCA2 will increase the level of G4 at telomeres. Therefore, it will be interesting to see whether the increase in telomere G4 is directly associated with the induction of BIR and ALT. In sum, our data suggest that BRCA2 maintains telomere replication homeostasis through binding to dynamic telomeric G4 structures and controls the accessibility of the machinery linked to telomere metabolism. 


\section{Methods}

\section{Purification of recombinant proteins}

BRCA2OB (2670-3184 a.a.) and POT1OB (11-278 a.a.) were inserted into pMAL-c5X with a $6 \times$ His-tag added at the C-terminus. They were expressed in E. coli and induced using 0.1 mM IPTG at $18^{\circ} \mathrm{C}$ overnight. Cells were lysed in lysis buffer $(50 \mathrm{mM}$ Tris- $\mathrm{HCl} \mathrm{pH} 7.5,150$ $\mathrm{mM} \mathrm{NaCl}, 2 \mathrm{mM}$-mercaptoethanol, $3 \mathrm{mM} \mathrm{MgCl} 2,10 \mathrm{mM}$ imidazole, 0.5\% NP-40, $1 \mathrm{mM}$ EDTA, and $1 \mathrm{mM}$ PMSF), followed by sonication. The lysate was centrifuged at 20,000 RPM for $1 \mathrm{~h}$, and the proteins were eluted using Ni-NTA. Mre11N (1-411 a.a.) were inserted in pET-28 (Novagen) and purified as described previously.

\section{Electrophoretic mobility shift assay (EMSA)}

$10 \mathrm{nM} \gamma-{ }^{32} \mathrm{P}$-end-labeled DNA probes were pre-incubated at $37^{\circ} \mathrm{C}$ for $15 \mathrm{~min}$ in EMSA buffer (50 mM Tris- $\mathrm{HCl} \mathrm{pH} 7.5,100 \mathrm{mM} \mathrm{KCl,} 2 \mathrm{mM} \beta$-mercaptoethanol, and $10 \%$ glycerol) and the purified protein was added. After incubation, the mixture was loaded onto a $5 \%$ polyacrylamide gel containing $0.5 \mathrm{X} \mathrm{TBE}$ buffer, and the gels were run at $10 \mathrm{~V} / \mathrm{cm}$ for $2 \mathrm{hr}$ at $4^{\circ} \mathrm{C}$. Gels were dried using a 583 Gel dryer (Bio-rad) and data captured using the Fuji FLA 7000 scanner. The signal intensity was quantified using Multi Gauge V3.0 software (Fujifilm, Tokyo, Japan). Data were analyzed by curve fitting and regression (Prism; GraphPad Software Inc., San Diego, CA) to determine the equilibrium dissociation constant, using the one site specific binding equation: $\mathrm{Y}=\mathrm{Bmax} * \mathrm{X} /\left(K_{d}+\mathrm{X}\right)(\mathrm{Bmax}=1$ set as a constraint $)$. Sequences of DNA probes used in EMSA are listed in Supplementary Table 1.

\section{Immunoprecipitation and DNA pull-down assay}


NFLAP-BRCA2 HeLa cells and HeLa cells were lysed in IP buffer [50 mM Tris-HCl pH 7.5, 10\% glycerol, $150 \mathrm{mM} \mathrm{NaCl}, 1 \mathrm{mM}$ DTT, $1 \mathrm{mM}$ EDTA, 0.2\% Triton X-100, $5 \mathrm{mM} \mathrm{NaF,} 200$ $\mu \mathrm{M}$ Na-orthovanadate and protease inhibitor cocktail (Roche) $]^{87}$, and sonication was performed. The lysates were incubated with $1 \mathrm{mM} \mathrm{MgCl}_{2}$ and benzonase for $1 \mathrm{~h}$ at $4{ }^{\circ} \mathrm{C}$ and centrifuged at $6000 \mathrm{RPM}$ for $10 \mathrm{~min}$. The supernatant was transferred to GFP-Trap Agarose (ChromoTek) and incubated overnight at $4{ }^{\circ} \mathrm{C}$. After washing the beads with IP buffer, they were incubated in IP buffer containing $15 \mathrm{mM} \mathrm{MgCl}_{2} \mathrm{IP}$, followed by incubation with $\gamma$ - ${ }^{32} \mathrm{P}$-end-labeled DNA probes in EMSA buffer. BRCA2 bound probes were eluted from the GFP-beads by boiling at $95{ }^{\circ} \mathrm{C}$, while unbound probes were washed out. The eluted probes were dot-blotted onto a positively charged nylon membrane (Amersham ${ }^{\mathrm{TM}} \mathrm{Hybond}^{\mathrm{TM}}-\mathrm{N}+$ ) and imaged as previously described.

\section{Single-molecule FRET DNA sample preparation}

DNA oligonucleotides for single-molecule experiments were purchased from Integrated DNA Technologies (IDT). The G4-forming DNA strands were amino-modified at the 3' end, while a short complementary strand for partial duplex (stem) formation was 5' amino-modified and 3' biotinylated for surface immobilization. Fluorophores ( $\mathrm{Cy} 3$ or $\mathrm{Cy} 5)$ were couple to DNA using NHS-ester conjugated dyes (GE Healthcare). G4 and partial duplex formation were induced by slowly annealing the DNA substrates from $95^{\circ} \mathrm{C}$ to room temperature in $250 \mathrm{mM}$ $\mathrm{KCl}, 25 \mathrm{mM}$ Tris-HCl $\mathrm{pH}$ 8.0. The sequences of DNA constructs, including biotin and/or amino modifications, are listed in Supplementary Table 2.

\section{Circular Dichroism (CD) Spectroscopy}

To measure the CD spectra, $10 \mu \mathrm{M}$ DNA probes were prepared by heating at $95^{\circ} \mathrm{C}$ for $10 \mathrm{~min}$ 
and cooling down to room temperature in $0 \sim 100 \mathrm{mM} \mathrm{KCl}, 50 \mathrm{mM}$ Tris- $\mathrm{HCl} \mathrm{pH}$ 7.5. The CD spectra were recorded on JASCO J-815 spectropolarimeter between 220-320 nm of wavelength using $1 \mathrm{~mm}$ path length quartz cuvette at $37^{\circ} \mathrm{C}$. Each $\mathrm{CD}$ spectroscopy measurement is the average of three scans with $200 \mathrm{~nm} / \mathrm{min}$ scanning speed. The sequences of DNA probes in CD are listed in Supplementary Table 1.

\section{Single-molecule FRET measurement and data analysis}

Single-molecule FRET experiments were performed using a home-built prism-type total internal reflection fluorescence microscope ${ }^{88}$. A single-molecule imaging chamber was assembled with a microscope quartz slide and glass coverslips that were coated with polyethylene glycol and surface-biotinylated. For all single-molecule imaging, biotinylated DNA constructs were immobilized on the chamber surface via the biotin-NeutrAvidin interaction and imaged with or without BRCA2OB at $37^{\circ} \mathrm{C}$ in imaging buffer [50 mM Tris$\mathrm{HCl}$ pH 7.9, $100 \mathrm{mM} \mathrm{KCl}, 20 \mathrm{mM} \mathrm{NaCl}, 1 \mathrm{mM}$ DTT, $0.1 \mathrm{mg} \mathrm{ml}^{-1} \mathrm{BSA}$, and 9\% (v/v) glycerol] with an oxygen scavenging system $\left[1 \mathrm{mg} \mathrm{ml}^{-1}\right.$ glucose oxidase (Sigma-Aldrich), $0.04 \mathrm{mg} \mathrm{ml}^{-1}$ catalase (Sigma-Aldrich), and 0.8\% (w/v) $\beta$-D-glucose (Sigma-Aldrich)] and a triplet quencher [ 3 mM Trolox (Acros Organics)] for enhanced photostability. Fluorescence signals from individual DNA molecules were recorded with a time window of $2 \mathrm{~s}$ and $200 \mathrm{~s}$ (100 ms of the acquisition time) in order to construct smFRET histograms and time trajectories, respectively, using a homemade C\# script. In the absence of BRCA2OB, images were collected for $\sim 20$ min intermittently, while the FRET histograms and time trajectories in the presence of BRCA2OB were obtained $5 \mathrm{~min}$ and $10 \mathrm{~min}$ after protein addition, respectively. For FRET histograms, green-red alternating laser excitation was introduced if necessary during data acquisition in order to focus on dual-labeled molecules. Data from over 2000 molecules were used to build each histogram, and fitted by multiple Gaussian curves; for histograms in the 
presence of BRCA2OB, the center FRET values of the UF, NP, and P conformational peaks were fixed to those obtained without the protein (or restricted to the protein-free values \pm 0.01 ). The relative population of each FRET state was quantified as the relative area under each Gaussian fit curve. For the transition-density and dwell-time analyses, individual time trajectories were categorized manually into docked or transitioning molecules with the criterion of whether the trajectory exhibits FRET transitions or not during the first 100-s data points. Then, fragments of the transitioning trajectories (from $>200$ molecules) were collected, and were fitted into 2-4 states applying the variational Bayesian method (vbFRET) ${ }^{89}$ to construct the transition density and dwell time plots. Data processing and analysis was performed using home-written IDL and MATLAB codes.

\section{Nuclease assay}

$10 \mathrm{nM}$ or $1 \mathrm{nM}$ of $\gamma_{-}{ }^{32} \mathrm{P}$-end-labeled DNA substrate was incubated in reaction buffer $[25 \mathrm{mM}$ MOPS (pH 7.0), $100 \mathrm{mM} \mathrm{KCl,} 1 \mathrm{mM} \mathrm{MnCl}_{2}$, and $1 \mathrm{mM} \mathrm{DTT}$ ] for $15 \mathrm{~min}$ at room temperature prior to reaction. Approximately 2.5 or $6 \mu \mathrm{M}$ of purified MRE1 $1 \mathrm{~N}$ was incubated at $37{ }^{\circ} \mathrm{C}$ for 60 min. Reactions with BRCA2OB and MRE11N were identical except $2.5 \mu \mathrm{M}$ or $5 \mu \mathrm{M}$ of $\mathrm{BRCA} 2 \mathrm{OB}$ was pre-incubated at $37^{\circ} \mathrm{C}$ for $15 \mathrm{~min}$ prior to addition of MRE11N. The reaction was stopped by adding $1 / 10$ volume of stop mixture (2\% SDS, $100 \mathrm{mM}$ EDTA, and $0.5 \mathrm{mg} / \mathrm{ml}$ proteinase $\mathrm{K}$ ), followed by a $5 \mathrm{~min}$ incubation at $37^{\circ} \mathrm{C}$. Reaction products were resolved on $15 \%$ denaturing polyacrylamide gels containing $7 \mathrm{M}$ Urea in $1 \mathrm{X}$ TBE buffer. Gels were run for $120 \mathrm{~min}$ at constant power and were fixed with buffer (30\% methanol, 5\% acetic acid, 5\% glycerol). The fixed gels were dried and imaged.

\section{Antibodies}


The following antibodies were purchased: anti-Mre11 (4895S; Cell signaling Technology), anti-phospho-Histone H2AX (ser139) (9718S; Cell Signaling Technology), and anti-TRF1 (TRF-78) (ab10579; Abcam).

\section{Immunofluorescence assay}

MEF cells were cultured with or without $1 \mu \mathrm{M}$ of $4-\mathrm{OHT}$ for $24 \mathrm{~h}$ and treated with lentiviral shMre11 for $48 \mathrm{~h}$. Production and infection of lentiviral shMre11 was performed as previously described ${ }^{82}$. MEFs were serum starved for $16 \mathrm{~h}$ after infection, then cultured in the absence or presence of $5 \mu \mathrm{M}$ pyridostatin (PDS) for $4 \mathrm{~h}$. The cells were grown on a coverslip and fixed with 4\% paraformaldehyde, permeabilized in PBS containing $0.5 \%$ Triton $\mathrm{X}-100(0.5 \%$ PBS-T) for $15 \mathrm{~min}$ at room temperature. The coverslips were blocked in $10 \%$ goat serum in $0.1 \%$ PBS-T for $2 \mathrm{~h}$ at room temperature followed by overnight incubation with primary antibodies in blocking solution at $4{ }^{\circ} \mathrm{C}$. The cells were incubated with secondary antibodies for $2 \mathrm{~h}$ at room temperature, and mounted with VECTASHIELD mounting medium containing DAPI (Vector Laboratories). Microscopy images were acquired using DeltaVision as described above.

\section{Acknowledgments}

We thank the members of Hyunsook Lee's lab and Seong Keun Kim's lab for technical assistance and critical discussions throughout the study (especially Jinho Park for helpful discussion on the smFRET results). This work was supported by the Samsung Science and Technology Foundation (SSTF-BA1301-05) to H.L. This work also was supported by the National Research Foundation of Korea (NRF) (NRF-2018R1A2B2001422 to S.K.K. and 
2020R1A5A1018081 to H.L.). K.S. was supported by the Global PhD Fellowship from the National Research Foundation of Korea (NRF-2018H1A2A1060095).

\section{Author contributions}

H.L conceived the study. J.L. designed and performed biochemical and cellular experiments, and was assisted by S.Y.J. for MRE11 nuclease assays. K.S. designed and performed singlemolecule assays and analyzed the data. H.L. and S.K.K. supervised the research. The manuscript was written and approved by all authors.

\section{Competing interests statement}

The authors declare no competing financial interests.

\section{References}

1. McEachern, M.J. \& Blackburn, E.H. A conserved sequence motif within the exceptionally diverse telomeric sequences of budding yeasts. Proc Natl Acad Sci US A 91, 3453-3457 (1994).

2. Meyne, J., Ratliff, R.L. \& Moyzis, R.K. Conservation of the human telomere sequence (TTAGGG)n among vertebrates. Proc Natl Acad Sci U S A 86, 7049-7053 (1989).

3. Hoogsteen, K. Crystal and Molecular Structure of a Hydrogen-Bonded Complex between 1-Methylthymine and 9-Methyladenine. Acta Crystallographica 16(9), 907$916(1963)$.

4. Gellert, M., Lipsett, M.N. \& Davies, D.R. Helix formation by guanylic acid. Proc Natl Acad Sci U S A 48, 2013-2018 (1962).

5. Zimmerman, S.B., Cohen, G.H. \& Davies, D.R. X-ray fiber diffraction and modelbuilding study of polyguanylic acid and polyinosinic acid. J Mol Biol 92, 181-192 (1975). 
6. Williamson, J.R. G-quartet structures in telomeric DNA. Annu Rev Biophys Biomol Struct 23, 703-730 (1994).

7. Wang, Y. \& Patel, D.J. Solution structure of a parallel-stranded G-quadruplex DNA. $J$ Mol Biol 234, 1171-1183 (1993).

8. Crabbe, L., Verdun, R.E., Haggblom, C.I. \& Karlseder, J. Defective telomere lagging strand synthesis in cells lacking WRN helicase activity. Science 306, 1951-1953 (2004).

9. Lin, W. et al. Mammalian DNA2 helicase/nuclease cleaves G-quadruplex DNA and is required for telomere integrity. EMBO J 32, 1425-1439 (2013).

10. Drosopoulos, W.C., Kosiyatrakul, S.T. \& Schildkraut, C.L. BLM helicase facilitates telomere replication during leading strand synthesis of telomeres. J Cell Biol 210, 191208 (2015).

11. Paeschke, K. et al. Telomerase recruitment by the telomere end binding protein-beta facilitates G-quadruplex DNA unfolding in ciliates. Nat Struct Mol Biol 15, 598-604 (2008).

12. Oganesian, L., Graham, M.E., Robinson, P.J. \& Bryan, T.M. Telomerase recognizes Gquadruplex and linear DNA as distinct substrates. Biochemistry 46, 11279-11290 (2007).

13. Wooster, R. et al. Identification of the breast cancer susceptibility gene BRCA2. Nature 378, 789-792 (1995).

14. Breast Cancer Linkage, C. Cancer risks in BRCA2 mutation carriers. J Natl Cancer Inst 91, 1310-1316 (1999).

15. Badie, S. et al. BRCA2 acts as a RAD51 loader to facilitate telomere replication and capping. Nat Struct Mol Biol 17, 1461-1469 (2010).

16. Min, J. et al. The breast cancer susceptibility gene BRCA2 is required for the maintenance of telomere homeostasis. J Biol Chem 287, 5091-5101 (2012).

17. Lekomtsev, S., Guizetti, J., Pozniakovsky, A., Gerlich, D.W. \& Petronczki, M. Evidence that the tumor-suppressor protein BRCA2 does not regulate cytokinesis in human cells. J Cell Sci 123, 1395-1400 (2010).

18. Choi, E. et al. BRCA2 fine-tunes the spindle assembly checkpoint through reinforcement of BubR1 acetylation. Developmental cell 22, 295-308 (2012).

19. Yang, $\mathrm{H}$. et al. BRCA2 function in DNA binding and recombination from a BRCA2DSS1-ssDNA structure. Science 297, 1837-1848 (2002).

20. Lei, M., Podell, E.R. \& Cech, T.R. Structure of human POT1 bound to telomeric singlestranded DNA provides a model for chromosome end-protection. Nat Struct Mol Biol 11, 1223-1229 (2004). 
21. Lei, M., Podell, E.R., Baumann, P. \& Cech, T.R. DNA self-recognition in the structure of Pot1 bound to telomeric single-stranded DNA. Nature 426, 198-203 (2003).

22. Lei, M., Baumann, P. \& Cech, T.R. Cooperative binding of single-stranded telomeric DNA by the Pot1 protein of Schizosaccharomyces pombe. Biochemistry 41, 1456014568 (2002).

23. Choi, E. et al. BRCA2 Fine-Tunes the Spindle Assembly Checkpoint through Reinforcement of BubR1 Acetylation. Dev Cell 22, 295-308 (2012).

24. Zhao, W. et al. Promotion of BRCA2-Dependent Homologous Recombination by DSS1 via RPA Targeting and DNA Mimicry. Mol Cell 59, 176-187 (2015).

25. Koirala, D. et al. A single-molecule platform for investigation of interactions between G-quadruplexes and small-molecule ligands. Nat Chem 3, $782-787$ (2011).

26. Rodriguez, R. et al. Small-molecule-induced DNA damage identifies alternative DNA structures in human genes. Nat Chem Biol 8, 301-310 (2012).

27. Zimmer, J. et al. Targeting BRCA1 and BRCA2 Deficiencies with G-QuadruplexInteracting Compounds. Mol Cell 61, 449-460 (2016).

28. McLuckie, K.I. et al. G-quadruplex DNA as a molecular target for induced synthetic lethality in cancer cells. J Am Chem Soc 135, 9640-9643 (2013).

29. Olivieri, M. et al. A Genetic Map of the Response to DNA Damage in Human Cells. Cell 182, 481-496 e21 (2020).

30. Hegyi, H. Enhancer-promoter interaction facilitated by transiently forming Gquadruplexes. Sci Rep 5, 9165 (2015).

31. Huppert, J.L. \& Balasubramanian, S. G-quadruplexes in promoters throughout the human genome. Nucleic Acids Res 35, 406-413 (2007).

32. Maizels, N. \& Gray, L.T. The G4 genome. PLoS Genet 9, e1003468 (2013).

33. Chambers, V.S. et al. High-throughput sequencing of DNA G-quadruplex structures in the human genome. Nat Biotechnol 33, 877-881 (2015).

34. Hansel-Hertsch, R. et al. G-quadruplex structures mark human regulatory chromatin. Nat Genet 48, 1267-1272 (2016).

35. Ambrus, A., Chen, D., Dai, J.X., Jones, R.A. \& Yang, D.Z. Solution structure of the biologically relevant g-quadruplex element in the human c-MYC promoter. implications for g-quadruplex stabilization. Biochemistry 44, 2048-2058 (2005).

36. Allshire, R.C., Dempster, M. \& Hastie, N.D. Human telomeres contain at least three types of G-rich repeat distributed non-randomly. Nucleic acids research 17, 4611-4627 (1989). 
37. Baird, D.M., Jeffreys, A. \& Royle, N. Mechanisms underlying telomere repeat turnover, revealed by hypervariable variant repeat distribution patterns in the human $\mathrm{Xp} / \mathrm{Yp}$ telomere. The EMBO journal 14, 5433-5443 (1995).

38. Baird, D.M., Coleman, J., Rosser, Z.H. \& Royle, N.J. High levels of sequence polymorphism and linkage disequilibrium at the telomere of 12q: implications for telomere biology and human evolution. The American Journal of Human Genetics 66, 235-250 (2000).

39. Coleman, J., Baird, D.M. \& Royle, N.J. The plasticity of human telomeres demonstrated by a hypervariable telomere repeat array that is located on some copies of 16p and 16q. Human molecular genetics 8, 1637-1646 (1999).

40. Lee, M. et al. Telomere extension by telomerase and ALT generates variant repeats by mechanistically distinct processes. Nucleic Acids Res 42, 1733-1746 (2014).

41. Rajavel, M. et al. Dynamic peptides of human TPP1 fulfill diverse functions in telomere maintenance. Nucleic Acids Res 44, 10467-10479 (2016).

42. Yagi, R., Miyazaki, T.\& Oyoshi, T. G-quadruplex binding ability of TLS/FUS depends on the $\beta$-spiral structure of the RGG domain. Nucleic acids research 46, 5894-5901 (2018).

43. González, V., Guo, K., Hurley, L. \& Sun, D. Identification and characterization of nucleolin as a c-myc G-quadruplex-binding protein. Journal of Biological Chemistry 284, 23622-23635 (2009).

44. McCormick-Graham, M. \& Romero, D.P. A single telomerase RNA is sufficient for the synthesis of variable telomeric DNA repeats in ciliates of the genus Paramecium. Mol Cell Biol 16, 1871-1879 (1996).

45. Greider, C.W. \& Blackburn, E.H. Identification of a specific telomere terminal transferase activity in Tetrahymena extracts. Cell 43, 405-413 (1985).

46. Henderson, E., Hardin, C.C., Walk, S.K., Tinoco, I., Jr. \& Blackburn, E.H. Telomeric DNA oligonucleotides form novel intramolecular structures containing guanineguanine base pairs. Cell 51, 899-908 (1987).

47. Sieverling, L. et al. Genomic footprints of activated telomere maintenance mechanisms in cancer. Nat Commun 11, 733 (2020).

48. Zaug, A.J., Podell, E.R. \& Cech, T.R. Human POT1 disrupts telomeric G-quadruplexes allowing telomerase extension in vitro. Proc Natl Acad Sci U S A 102, 10864-10869 (2005).

49. Wang, Y. \& Patel, D.J. Solution structure of the human telomeric repeat d[AG3(T2AG3)3] G-tetraplex. Structure 1, 263-282 (1993).

50. Parkinson, G.N., Lee, M.P. \& Neidle, S. Crystal structure of parallel quadruplexes from human telomeric DNA. Nature 417, 876-880 (2002). 
51. Dai, J., Carver, M., Punchihewa, C., Jones, R.A. \& Yang, D. Structure of the Hybrid-2 type intramolecular human telomeric G-quadruplex in $\mathrm{K}+$ solution: insights into structure polymorphism of the human telomeric sequence. Nucleic Acids Res 35, 49274940 (2007).

52. Ambrus, A. et al. Human telomeric sequence forms a hybrid-type intramolecular Gquadruplex structure with mixed parallel/antiparallel strands in potassium solution. Nucleic Acids Res 34, 2723-2735 (2006).

53. Phan, A.T., Kuryavyi, V., Luu, K.N. \& Patel, D.J. Structure of two intramolecular Gquadruplexes formed by natural human telomere sequences in $\mathrm{K}+$ solution. Nucleic Acids Res 35, 6517-6525 (2007).

54. Tippana, R., Xiao, W. \& Myong, S. G-quadruplex conformation and dynamics are determined by loop length and sequence. Nucleic Acids Res 42, 8106-81014 (2014).

55. Cheng, M. et al. Loop permutation affects the topology and stability of G-quadruplexes. Nucleic Acids Res 46, 9264-9275 (2018).

56. Burge, S., Parkinson, G.N., Hazel, P., Todd, A.K. \& Neidle, S. Quadruplex DNA: sequence, topology and structure. Nucleic Acids Res 34, 5402-5415 (2006).

57. Sattin, G. et al. Conformation and stability of intramolecular telomeric G-quadruplexes: sequence effects in the loops. PLoS One 8, e84113 (2013).

58. Dhakal, S. et al. Structural and mechanical properties of individual human telomeric Gquadruplexes in molecularly crowded solutions. Nucleic Acids Res 41, 3915-3923 (2013).

59. Lee, J.Y., Okumus, B., Kim, D.S. \& Ha, T. Extreme conformational diversity in human telomeric DNA. Proc Natl Acad Sci US A 102, 18938-18943 (2005).

60. Ying, L., Green, J.J., Li, H., Klenerman, D. \& Balasubramanian, S. Studies on the structure and dynamics of the human telomeric $\mathrm{G}$ quadruplex by single-molecule fluorescence resonance energy transfer. Proc Natl Acad Sci U S A 100, 14629-14634 (2003).

61. Ray, S., Bandaria, J.N., Qureshi, M.H., Yildiz, A. \& Balci, H. G-quadruplex formation in telomeres enhances POT1/TPP1 protection against RPA binding. Proc Natl Acad Sci U S A 111, 2990-2995 (2014).

62. Tippana, R., Hwang, H., Opresko, P.L., Bohr, V.A. \& Myong, S. Single-molecule imaging reveals a common mechanism shared by G-quadruplex-resolving helicases. Proc Natl Acad Sci U S A 113, 8448-8453 (2016).

63. Long, X. \& Stone, M.D. Kinetic partitioning modulates human telomere DNA Gquadruplex structural polymorphism. PLoS One 8, e83420 (2013).

64. Rocca, R. et al. Folding intermediate states of the parallel human telomeric Gquadruplex DNA explored using Well-Tempered Metadynamics. Scientific reports 10, $1-11(2020)$. 
65. Stadlbauer, P. et al. Parallel G-triplexes and G-hairpins as potential transitory ensembles in the folding of parallel-stranded DNA G-Quadruplexes. Nucleic Acids Res 47, 7276-7293 (2019).

66. Mashimo, T., Yagi, H., Sannohe, Y., Rajendran, A. \& Sugiyama, H. Folding pathways of human telomeric type-1 and type-2 G-quadruplex structures. J Am Chem Soc 132, 14910-14918 (2010).

67. Zhang, A.Y. \& Balasubramanian, S. The kinetics and folding pathways of intramolecular G-quadruplex nucleic acids. J Am Chem Soc 134, 19297-19308 (2012).

68. Gray, R.D., Trent, J.O. \& Chaires, J.B. Folding and unfolding pathways of the human telomeric G-quadruplex. J Mol Biol 426, 1629-1650 (2014).

69. Limongelli, V. et al. The G-triplex DNA. Angew Chem Int Ed Engl 52, 2269-2273 (2013).

70. Cerofolini, L. et al. G-triplex structure and formation propensity. Nucleic Acids Res $\mathbf{4 2}$, 13393-13404 (2014).

71. Li, W., Hou, X.M., Wang, P.Y., Xi, X.G. \& Li, M. Direct measurement of sequential folding pathway and energy landscape of human telomeric G-quadruplex structures. $J$ Am Chem Soc 135, 6423-6426 (2013).

72. Koirala, D. et al. Long-loop G-quadruplexes are misfolded population minorities with fast transition kinetics in human telomeric sequences. J Am Chem Soc 135, 2235-2241 (2013).

73. Hou, X.M. et al. Involvement of G-triplex and G-hairpin in the multi-pathway folding of human telomeric G-quadruplex. Nucleic Acids Res 45, 11401-11412 (2017).

74. Lim, K.W. et al. Structure of the human telomere in K+ solution: a stable basket-type G-quadruplex with only two G-tetrad layers. J Am Chem Soc 131, 4301-4309 (2009).

75. Zhang, Z., Dai, J., Veliath, E., Jones, R.A. \& Yang, D. Structure of a two-G-tetrad intramolecular G-quadruplex formed by a variant human telomeric sequence in $\mathrm{K}+$ solution: insights into the interconversion of human telomeric G-quadruplex structures. Nucleic acids research 38, 1009-1021 (2010).

76. Lee, H. Cycling with BRCA2 from DNA repair to mitosis. Exp Cell Res 329, 78-84 (2014).

77. Schlacher, K. et al. Double-strand break repair-independent role for BRCA2 in blocking stalled replication fork degradation by MRE11. Cell 145, 529-542 (2011).

78. Ghosal, G. \& Muniyappa, K. The characterization of Saccharomyces cerevisiae Mre11/Rad50/Xrs2 complex reveals that Rad50 negatively regulates Mre11 endonucleolytic but not the exonucleolytic activity. J Mol Biol 372, 864-882 (2007).

79. Park, Y.B., Chae, J., Kim, Y.C. \& Cho, Y. Crystal structure of human Mre11: understanding tumorigenic mutations. Structure 19, 1591-1602 (2011). 
80. Lim, H.S., Kim, J.S., Park, Y.B., Gwon, G.H. \& Cho, Y. Crystal structure of the Mre11Rad50-ATPgammaS complex: understanding the interplay between Mre11 and Rad50. Genes Dev 25, 1091-1104 (2011).

81. Hwang, H. et al. Telomeric overhang length determines structural dynamics and accessibility to telomerase and ALT-associated proteins. Structure 22, 842-853 (2014).

82. Kwon, M.S. et al. Brca2 abrogation engages with the alternative lengthening of telomeres via break-induced replication. FEBS J 286, 1841-1858 (2019).

83. Dupre, A. et al. A forward chemical genetic screen reveals an inhibitor of the Mre11Rad50-Nbs1 complex. Nat Chem Biol 4, 119-125 (2008).

84. Smith, J.S. et al. Rudimentary G-quadruplex-based telomere capping in Saccharomyces cerevisiae. Nat Struct Mol Biol 18, 478-485 (2011).

85. Tsai, Y.C., Qi, H. \& Liu, L.F. Protection of DNA ends by telomeric 3' G-tail sequences. J Biol Chem 282, 18786-18792 (2007).

86. Lee, J.J., Lee, J. \& Lee, H. Alternative paths to telomere elongation. Semin Cell Dev Biol 113, 88-96 (2021).

87. Thorslund, T. et al. The breast cancer tumor suppressor BRCA2 promotes the specific targeting of RAD51 to single-stranded DNA. Nat Struct Mol Biol 17, 1263-1265 (2010).

88. Sung, K., Park, J., Kim, Y., Lee, N.K. \& Kim, S.K. Target Specificity of Cas9 Nuclease via DNA Rearrangement Regulated by the REC2 Domain. J Am Chem Soc 140, 77787781 (2018).

89. Bronson, J.E., Fei, J., Hofman, J.M., Gonzalez, R.L., Jr. \& Wiggins, C.H. Learning rates and states from biophysical time series: a Bayesian approach to model selection and single-molecule FRET data. Biophys J 97, 3196-3205 (2009).

90. Jonkers, J. et al. Synergistic tumor suppressor activity of BRCA2 and p53 in a conditional mouse model for breast cancer. Nat Genet 29, 418-425 (2001). 


\section{Figure legends}

\section{Fig. 1. The BRCA2 OB-fold domain binds to telomeric G-quadruplexes.}

(a) Schematic illustration of human BRCA2. BRC repeats, DNA-binding domain (DBD), OB folds, and the nuclear localization signal (NLS) are marked. Recombinant BRCA2 OBfold (2670-3184; BRCA2OB), tagged with N-terminal MBP was purified (SDS-PAGE gel at right). (b) Electrophoresis Mobility Shift Assay (EMSA) was used to assess binding of recombinant BRCA2OB to telomere repeats. TelG5, G-rich single-stranded (GGTTAG)5 repeats; TelC5, C-rich strand complementary to TelG5; TelG5TelC5, double-stranded. ${ }^{32} \mathrm{P}-$ labeled hot probe is marked with asterisk(*). Arrow marks the BRCA2OB-bound hot oligonucleotide. (c) Increasing concentrations of BRCA2OB were incubated with TelG5 (lanes 2 to 4). Binding specificity was confirmed using competition with 1,000X excess of unlabeled TelG5 (+, Cold TelG5, lane 5) and Poly(dI-dC) for negative control (lane 6). Single-stranded (ss) telomeric repeat oligonucleotides of increasing length (TelG3 - TelG6, see illustration at the top) were incubated with (+) or without (-) BRCA2OB and subjected to EMSA. The binding of BRCA2OB to ss-telomere repeats was detected from TelG5 and TelG6. (e) BRCA2OB specifically binds to TelG5, which forms the G-quadruplex (G4) structure, but not to the single-stranded variant (TelG5GAG). (f) A G4 ligand PDS challenges the binding of BRCA2OB to G4 in a dose-dependent manner. EMSA analyses were performed in the presence of $100 \mathrm{mM} \mathrm{KCl}$.

\section{Fig. 2. Binding profile of BRCA2 to G4 telomere.}

(a) TelG5, TelG5TTT, and TelG5T2G4 probes $(10 \mathrm{nM})$ were incubated with BRCA2OB at the following concentrations: 0 (lane 1), 5nM (lane 2), 10nM (lane 3), 20nM (lane 4), 50nM (lane 5), 100nM (lane 6), 200nM (lane 7), 500nM (lane 8), and 1000nM (lane 9) (left). The $K_{d}$ 
values were analyzed by curve-fitting using nonlinear regression (mean \pm s.d.) (Right). TelG5, $K_{d}=4,466 \mathrm{nM}$; TelG5TTT, $K_{d}=456.0 \mathrm{nM}$; TelG5T2G4, $K_{d}=1.392 \mu \mathrm{M} . \quad$ (b) BRCA2OB and POT1OB were subjected to EMSA analysis with variant G4-forming telomere repeats (TVRs); TelG5, TelG5TTT, TelG5T2G4, and TelG5GAG (non-G4). EMSA analysis was performed in the presence of $100 \mathrm{mM} \mathrm{KCl}$. Human POT1 and the recombinant POT1 OBfold domain (POT1OB), purified from E. coli, are illustrated at top right. The OB fold domains and TPP1 binding domain are marked. The bar graph represents relative binding intensity (mean \pm s.e.m.). (c,d) Binding properties of full-length BRCA2 with TVRs (c) Schematic representation of the assay. EGFP-tagged full-length BRCA2 was immunoprecipitated from NFLAP-BRCA2 HeLa cells ${ }^{17,87}$ and incubated with labeled probes in the presence of $100 \mathrm{mM}$ KCl. Anti-GFP antibody was employed to pull down NFLAP-BRCA2 from the cell lysate. Unbound probes were washed out and NFLAP-BRCA2-bound probes, detected by anti-GFP antibodies, were eluted and subsequently dot blotted. (d) Dot blots showing the telomere binding properties of full-length BRCA2. HeLa cell lysate served as negative control. Five percent out of the total labeled probes were loaded as controls. Bar graph indicating the relative binding intensity of full-length BRCA2 to DNA probes (mean \pm s.e.m.).

\section{Fig. 3. Direct observation of the BRCA2OB-G4 telomere interaction at the single-}

\section{molecule level.}

(a) Schematic of the single-molecule FRET assay with dual-labeled TelG5 probes and recombinant BRCA2OB. Cartoons showing G4 conformational dynamics between the unfolded (UF), non-parallel (NP), and parallel (P) folded conformations, and BRCA2OBbound (BD) state. (b) CD (circular dichroism) spectra of the TelG5 in no salt (black), 10 $\mathrm{mM} \mathrm{KCl}$ (yellow, dashed), and $100 \mathrm{mM} \mathrm{KCl}$ (orange). (c) An smFRET histogram of TelG5 alone with three Gaussian fits (red) of the UF, NP, and P conformational peaks in the 
presence of $100 \mathrm{mM} \mathrm{KCl}$. The blue dashed curve represents the sum of the Gaussian fits. (d) A representative time trajectory of the TelG5 molecule with $100 \mathrm{mM} \mathrm{KCl}$ exhibiting dynamic transitions between UF, NP, and P conformations. (e) An smFRET histogram of TelG5 incubated with $1 \mu \mathrm{M}$ of BRCA2OB and $100 \mathrm{mM} \mathrm{KCl}$. The BRCA2OB-bound (BD) state is indicated by the black curve filled with semi-transparent blue. (f) A representative time trajectory of TelG5 in the presence of BRCA2OB and $100 \mathrm{mM} \mathrm{KCl}$. The BRCA2OBbound (BD) state was observed upon addition of BRCA2OB (orange broken line).

\section{Fig. 4. Conformational dynamics of the telomeric G4 determines the BRCA2 binding}

(a) smFRET histograms of the four G4 substrates (TelG5TAA, TelG5, TelG5TTT, and TelG5TT) with Gaussian fits color-coded as in Figure 3c. (b) smFRET histograms of the four G4 constructs upon incubation with $100 \mathrm{nM}$ BRCA2OB with Gaussian fits colored as in Figure 3e. (c) Average dwell time for UF (gray), NP (green), and P (red) conformations (mean \pm s.d.). (d) Transition density plots showing relative abundance of each transition (i.e., $\mathrm{UF} \rightarrow \mathrm{NP}, \mathrm{NP} \rightarrow \mathrm{UF}, \mathrm{UF} \rightarrow \mathrm{P}, \mathrm{P} \rightarrow \mathrm{UF}, \mathrm{NP} \rightarrow \mathrm{P}$, and $\mathrm{P} \rightarrow \mathrm{NP}$ ). (e) A representative time trajectory of the TelG5TTT molecule undergoing direct transitions between the NP and P conformations. (f) A comparative plot between the density of the NP-P direct transition (mean \pm s.d.) measured by the smFRET assays (blue bars, left axis) and relative binding of $\mathrm{BRCA} 2 \mathrm{OB}$ (mean \pm s.e.m.) quantified from the EMSA results (gray bars, right axis). (g) A molecular model of the BRCA2-G4 interaction. BRCA2 selectively binds to intermediates during structural rearrangement between the NP and P conformations. All smFRET analyses were performed in the presence of $100 \mathrm{mM} \mathrm{KCl}$.

Fig. 5. BRCA2 recognizes G-triplex-derived intermediates. (a) An experimental scheme to observe BRCA2OB binding towards the dual-labeled TelG5-G3 construct that mimics the 
G3 intermediate structure. smFRET histograms for (b) TelG5-G3 and (c) TelG5TTT-G3; DNA alone (top) and upon incubation with $1 \mu \mathrm{M}$ BRCA2OB (bottom) with Gaussian fits (red). BRCA2OB-interacting states are filled in semi-transparent blue. Blue dashed line is the sum of the multiple Gaussian curves. (d) A schematic representation (left) of the molecular interaction between BRCA2OB and G4 structures during the direct NP-P transition. For reference, smFRET histograms of TelG5 (middle) and TelG5TTT (right) in the presence of $1 \mu \mathrm{M}$ BRCA2OB from Fig. 3e and Supplementary Fig. S8, respectively, are shown (filled with gray). (e) EMSA to assess BRCA2OB binding to TelG5-G3, compared to TelG5 and TelG5TTT. TelG5GAG was employed as a negative control. Results are from a same gel. (f) A model for BRCA2 binding to the G3 and G3:G4 intermediates during G4 conformational interconversions. Two possible G3:G4 conformations and one representative G3 structure that might form during the NP-P rearrangement without complete unfolding are shown. BRCA2 recognizes and binds to G3 intermediates, which reduce folding into G4.

\section{Fig. 6. MRE11 resects G4 telomere}

(a) Schematic representation of human MRE11. The functional domains are marked. Recombinant hMRE11 core (1-441; MRE11N, underlined) was purified, using a previously published method ${ }^{79}$ (SDS-PAGE gel on the right), and employed in the nuclease assay. (b, c, d, e) Denaturing PAGE gels showing nuclease activity of MRE11N (lane 2-3) and the effect of BRCA2OB towards various telomere G4 and G3 variants (lane 4-5). $2.5 \mu \mathrm{M}$ or 6 $\mu \mathrm{M}$ of MRE11N was incubated with indicated DNA substrates: (b) TelG5-G3; (c) TelG5; (d) TelG5TTT; (e) TelG3GGG for nuclease assay. Increasing concentrations of BRCA2OB were employed to assess the inhibition of MRE11-mediated resection. 


\section{Fig. 7. Absence of Brca2 results in failure to protect MRE11-mediated resection of telomeric G4.}

(a) Schematic representation of the experimental design using TBI mouse fibroblasts. TBI fibroblasts were generated by immortalizing the telomerase-null $\left(\mathrm{mTR}^{-/-}\right) ; \mathrm{BrCa}^{\mathrm{Fl1} / \mathrm{Fl1}} ; \mathrm{Cre}$ $E R^{\mathrm{TM}} \mathrm{MEFs}^{90}$ with the introduction of SV40 $\mathrm{LT}^{82}$. Brca2 depletion was induced by tamoxifen (4-OHT) treatment. Mre11 depletion was achieved by transduction of lentiviralshMre11 (shMre11). The G4 stabilizer PDS was treated with the addition of 16\% FBS post $16 \mathrm{~h}$ of serum starvation. Cells were fixed for immunostaining $4 \mathrm{~h}$ after addition of serum to enrich S phase cells ${ }^{82}$. (b) Telomere damage in TBI fibroblasts was analyzed by immunostaining with anti-TRF1 antibody (red) and anti- $\gamma$-H2AX antibody (green) in the presence $(-4-\mathrm{OHT})$ or absence of Brca2 (+4-OHT). The effects of Mre11 depletion $(+$ shMre11) and that of G4 stabilizer addition (+PDS) were scored in the presence or absence of Brca2. Arrow points to the merged yellow foci representing damaged telomere. White scale bar, $5 \mu \mathrm{m}$. (c) The number of damaged telomeres per cell measured from (b). The number of $\gamma$-H2AX-positive telomeres per cell was counted and presented as bar graphs (mean \pm s.e.m.). The number of cells analyzed for each experiment: lane 1 (no treatment), $\mathrm{n}=$ 126; +shMre11, $\mathrm{n}=129 ;+$ PDS, $\mathrm{n}=129 ;+$ PDS +shMre11, $\mathrm{n}=131 ;+4-\mathrm{OHT}, \mathrm{n}=121 ;+4-$ OHT +shMre11, n = 92; +4-OHT +PDS, $\mathrm{n}=130 ;+4-\mathrm{OHT}+\mathrm{PDS}+$ shMre11, $\mathrm{n}=137$. (d) Western blot analysis to assess the efficiency of Brca2 depletion and Mre11 after 4-OHT treatment or shMrel1 transduction. The same blot was reprobed with anti-actin as a loading control. (e) Model for how BRCA2 and MRE11 work with telomeric G4 in replication homeostasis. G4 can be formed at lagging-strand telomeres during replication. Telomeric G4 exhibits highly dynamic structural transitions between P and NP-folded and UF-ssDNA, during which G3-derived intermediates can be formed transiently. In the absence of BRCA2, MRE11 resects telomeric G4. In the presence of BRCA2, BRCA2 identifies and 
binds to the G3-derived intermediate structures, thereby protecting telomeres from attack by MRE11. BRCA2 binding to G3-derived intermediates may facilitate the recruitment of a helicase $\mathrm{X}$, leading to the progression of lagging-strand telomere synthesis. 
Figure 1

a

Human BRCA2(3419a.a)

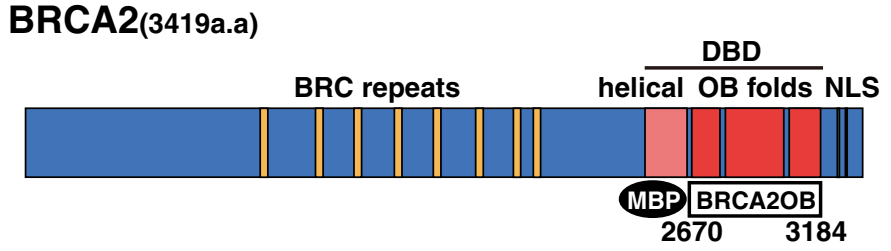

$\mathrm{kDa}$

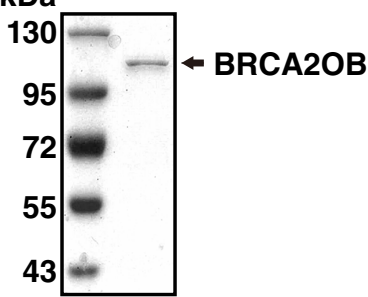

b

G-rich* G-rich*

G-rich C-rich C-rich* C-rich* BRCA2OB $\frac{\text { TelG5 }^{\star}}{-+} \frac{\begin{array}{l}\text { TelG5 } \\ \text { TelC5 }\end{array}}{-++} \frac{\text { TelC5 }^{*}}{-+} \frac{\begin{array}{l}\text { TelG5 } \\ \text { TelC5* }\end{array}}{-\quad+}$

C $\begin{array}{llllllll}\text { BRCA2OB } & 0 & 0.1 & 0.2 & 0.5 & 0.5 & 0.5 & (\mu \mathrm{M})\end{array}$ Cold TelG5 - $-\quad-\quad-1,000 \mathrm{x}-$ Poly(dl-dC)
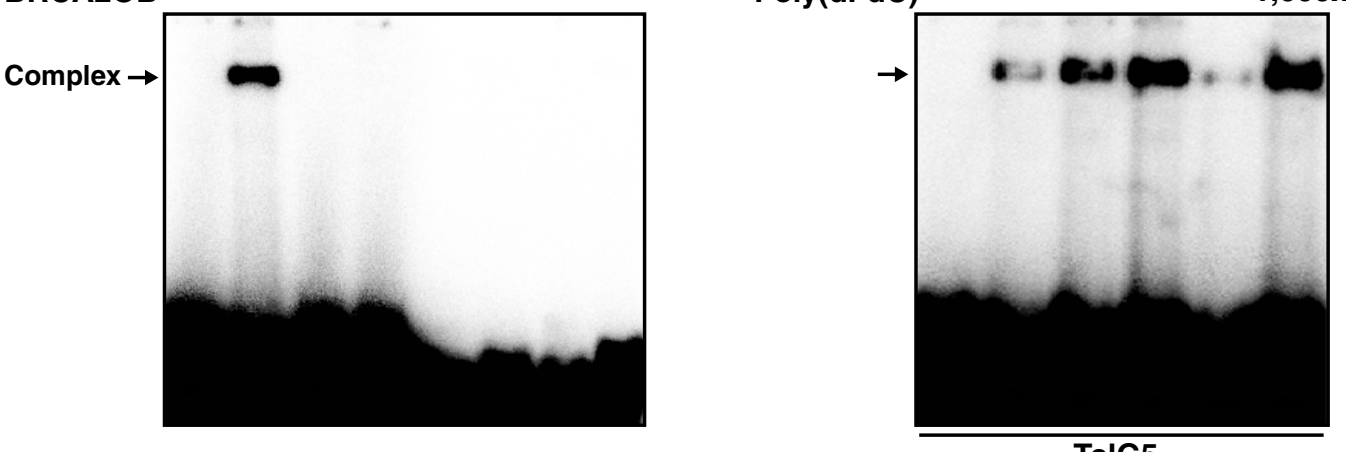

TelG5

d

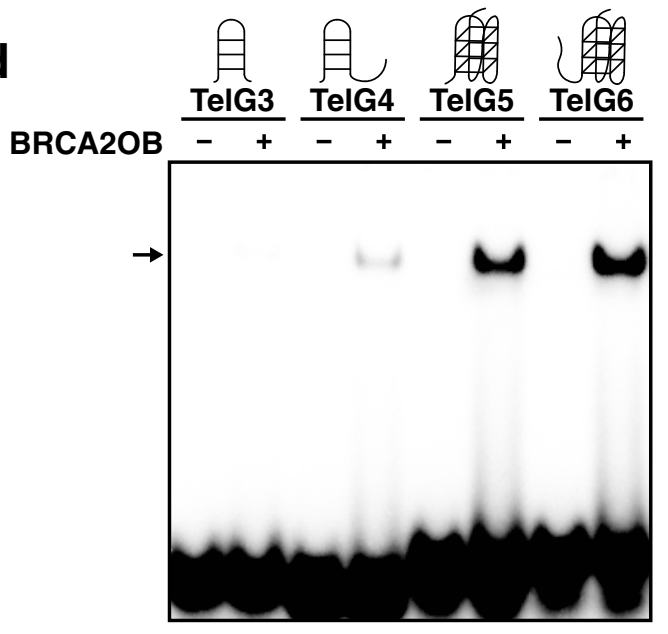

e

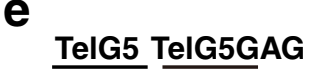

f

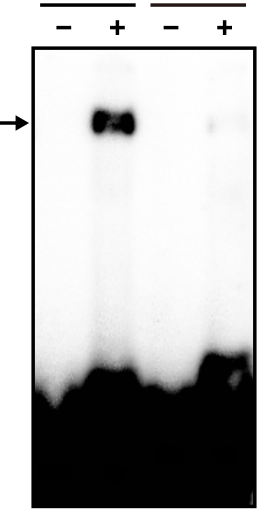

f \begin{tabular}{cccc}
\multicolumn{4}{c}{$\operatorname{PDS}(\mathrm{nM})$} \\
\hline 0 & 3 & 10 & 30 \\
+ & + & + & +
\end{tabular}

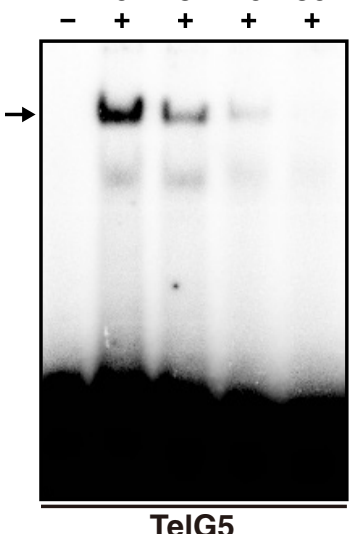


Figure 2

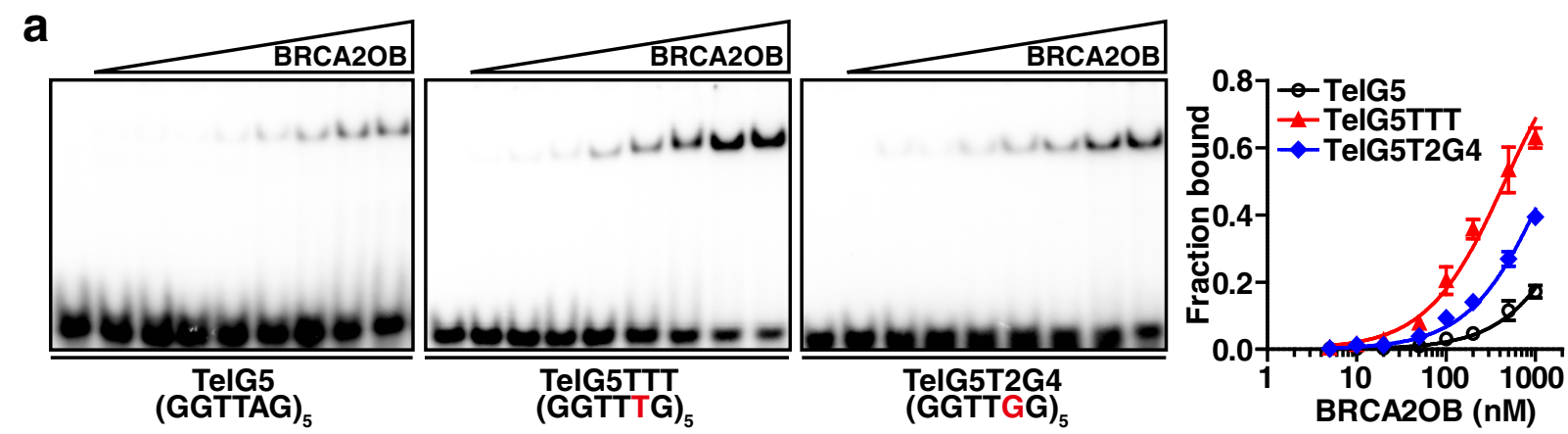

b

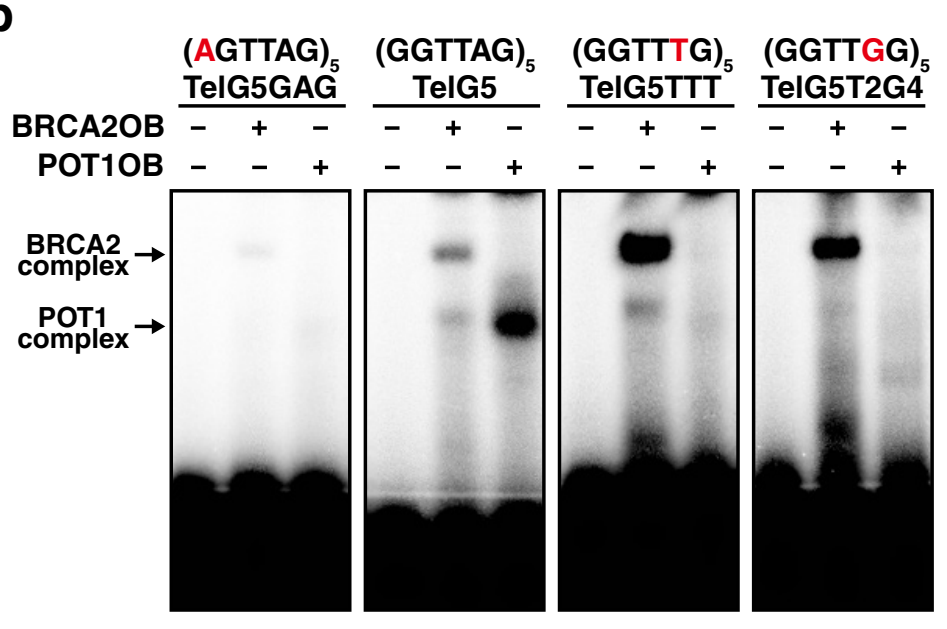

Human POT1(634a.a)
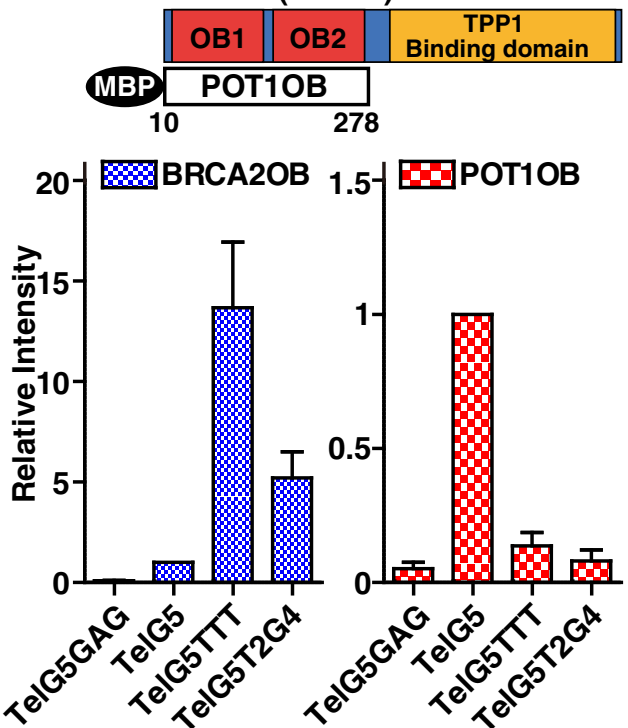

C

EGFP

NFLAP-BRCA2 ${ }^{\mathcal{G} F P}$-antibody cell lysate

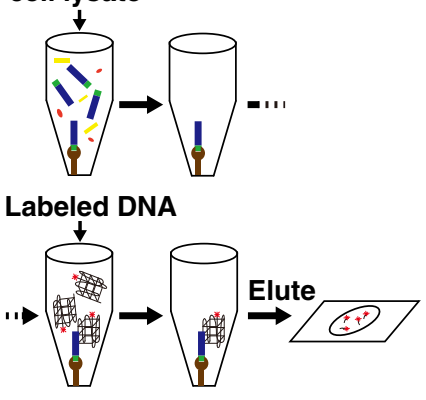

d

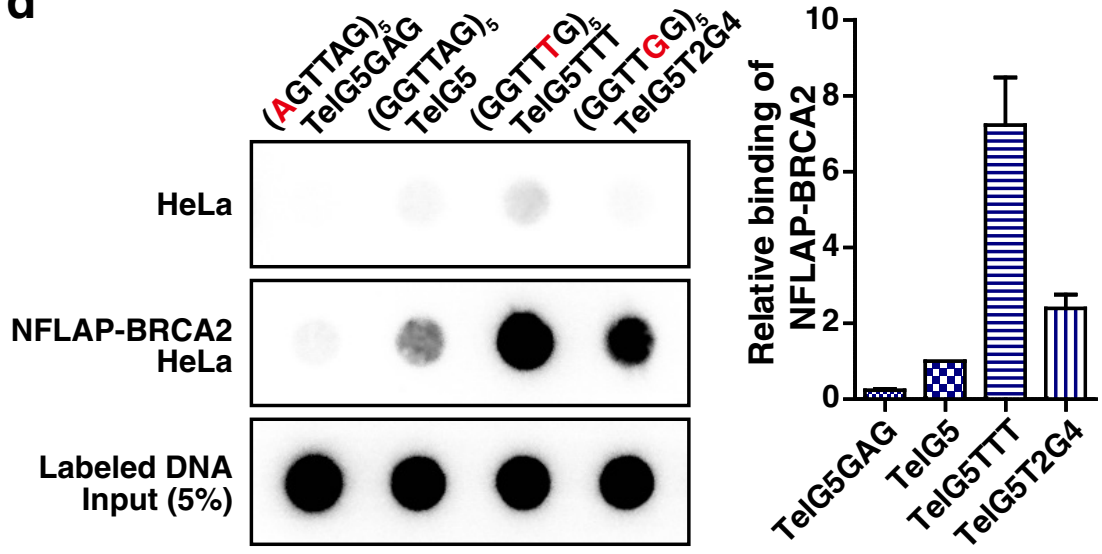


Figure 3
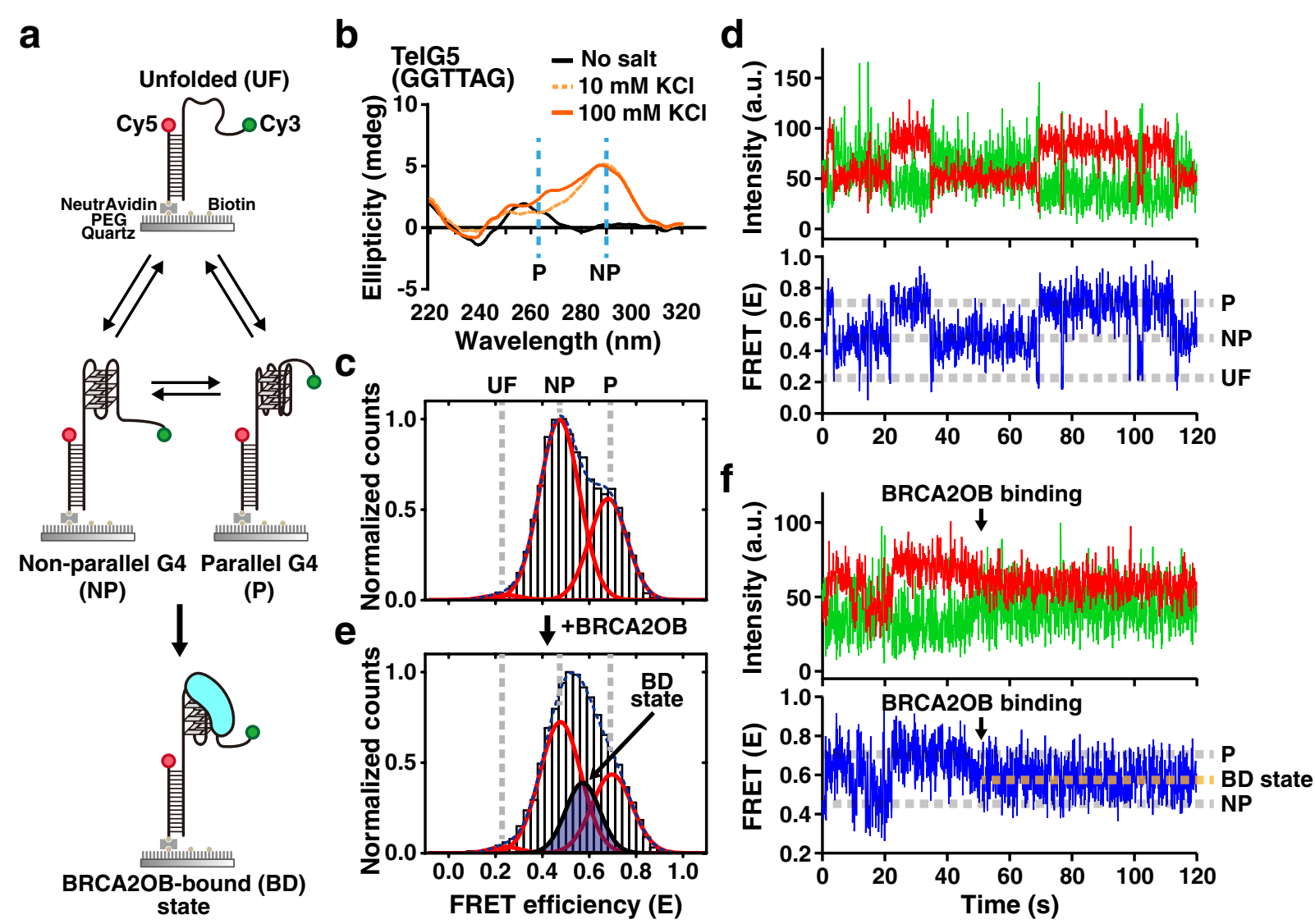
Figure 4

a
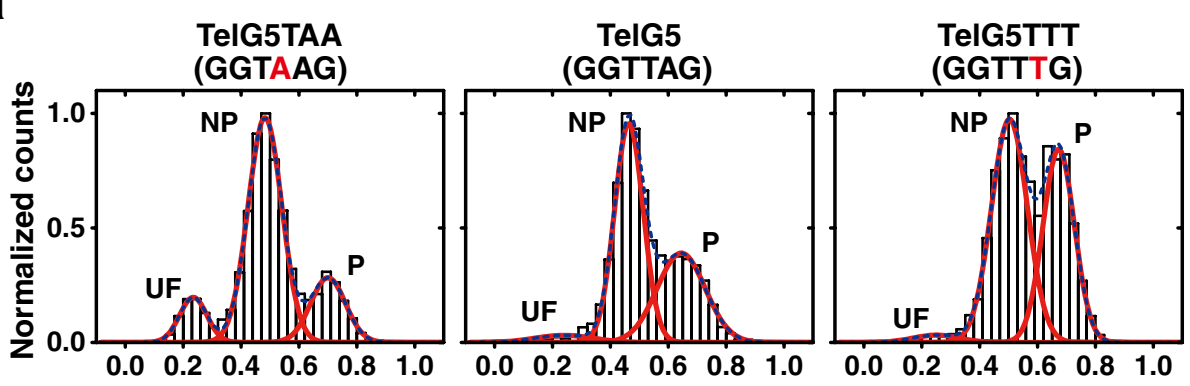

TelG5TT

(GGTTG)

FRET efficiency (E)

b

$\checkmark$ +ВRCA2OB
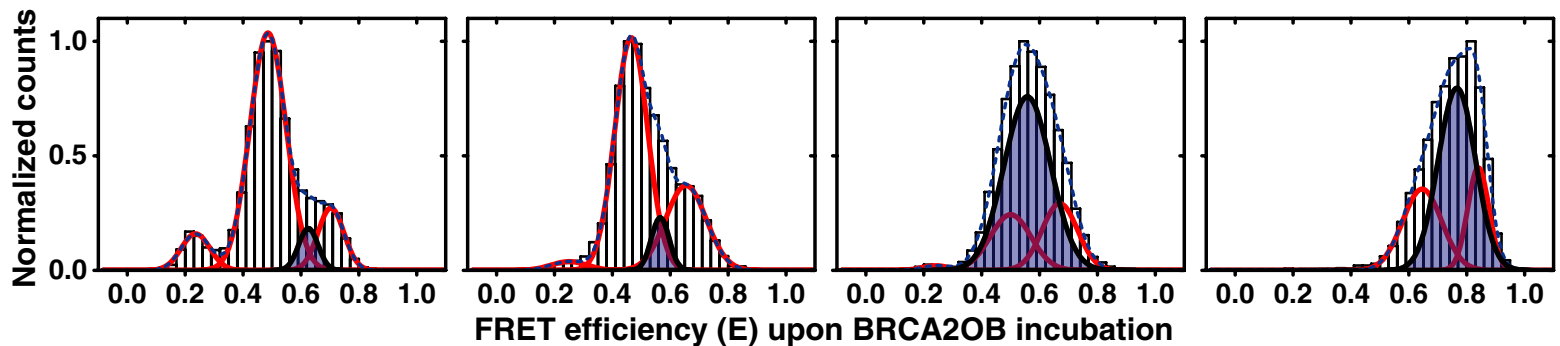

C

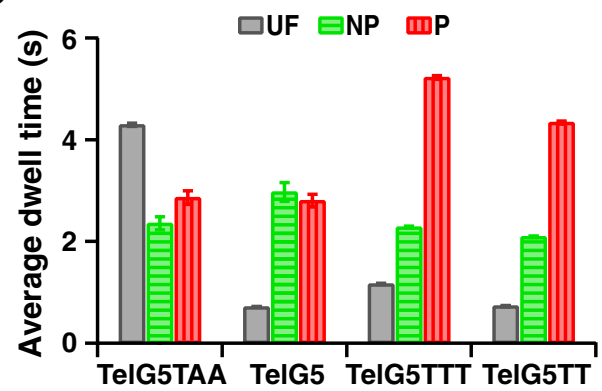

d
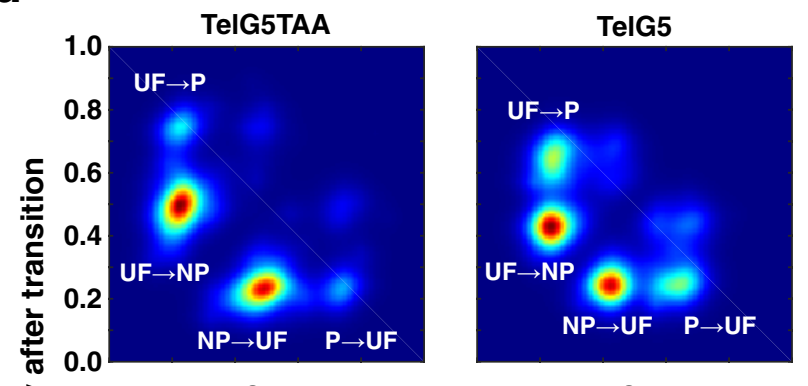

ठे 1.0 TelG5TTT

e
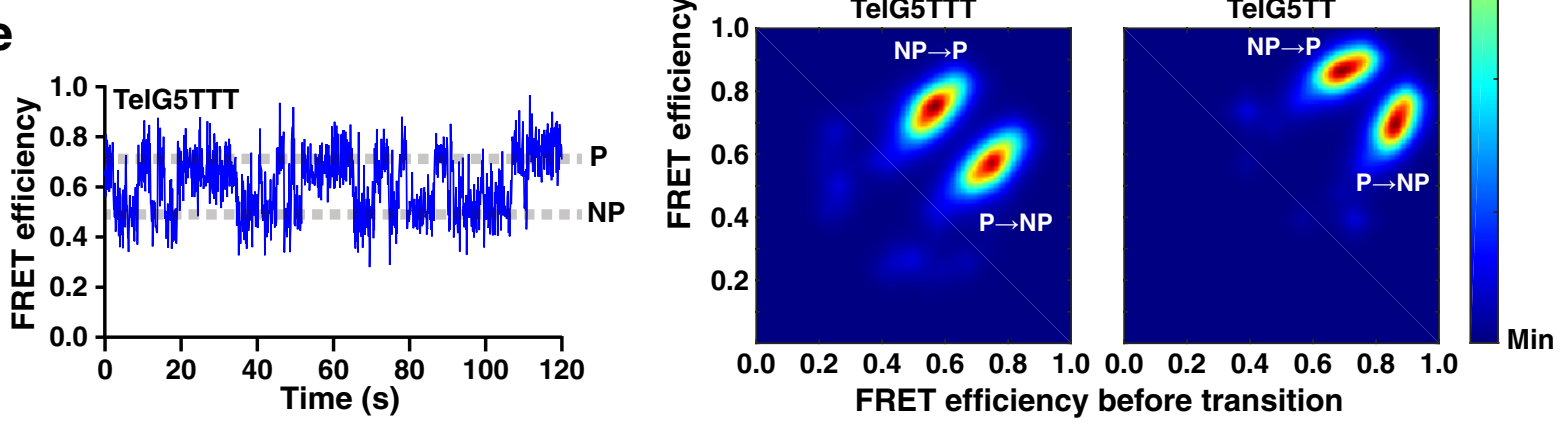

f

g
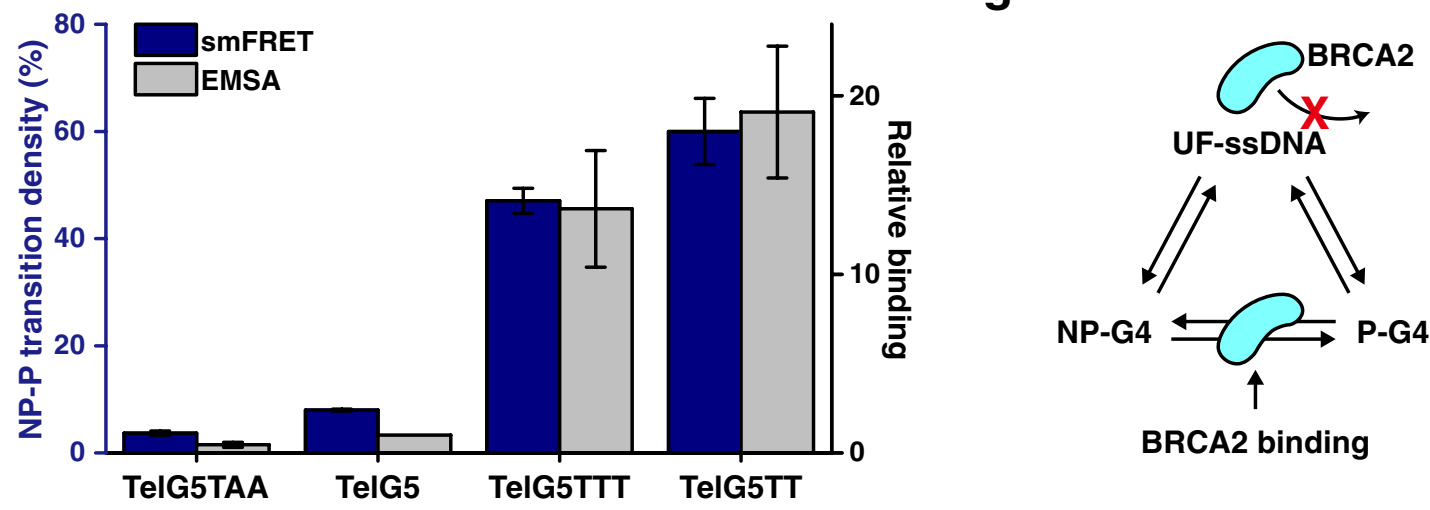
Figure 5

a

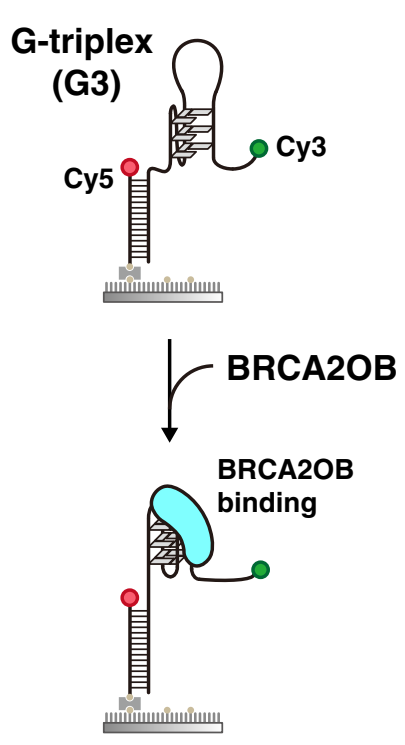

d

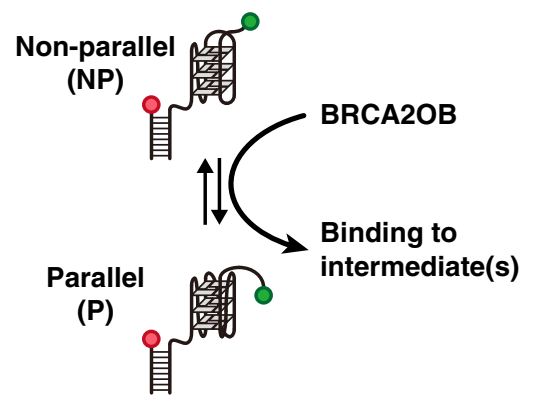

e

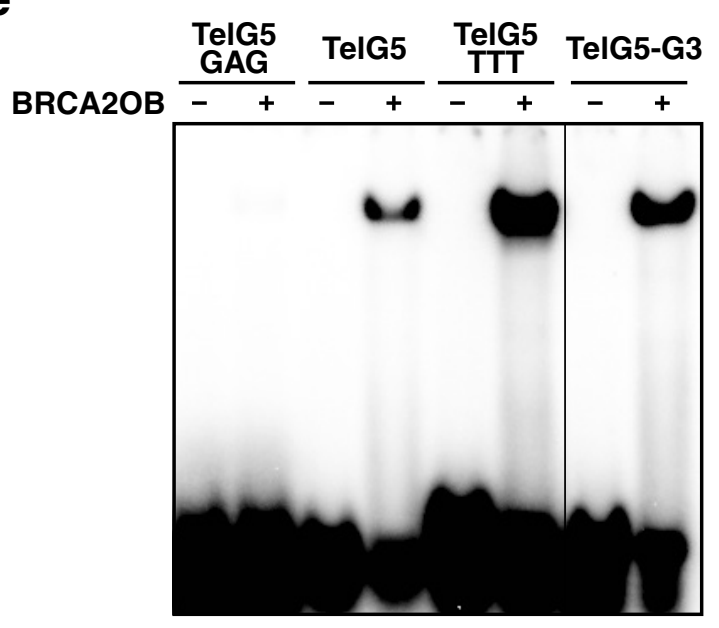

TelG5-G3

C

TeIG5TTT-G3

(GGTTAG) ${ }_{3}$-(TGTTAG)-(GGTTAG) (GGTTTG) ${ }_{3}$-(TGTTTG)-(GGTTTG)
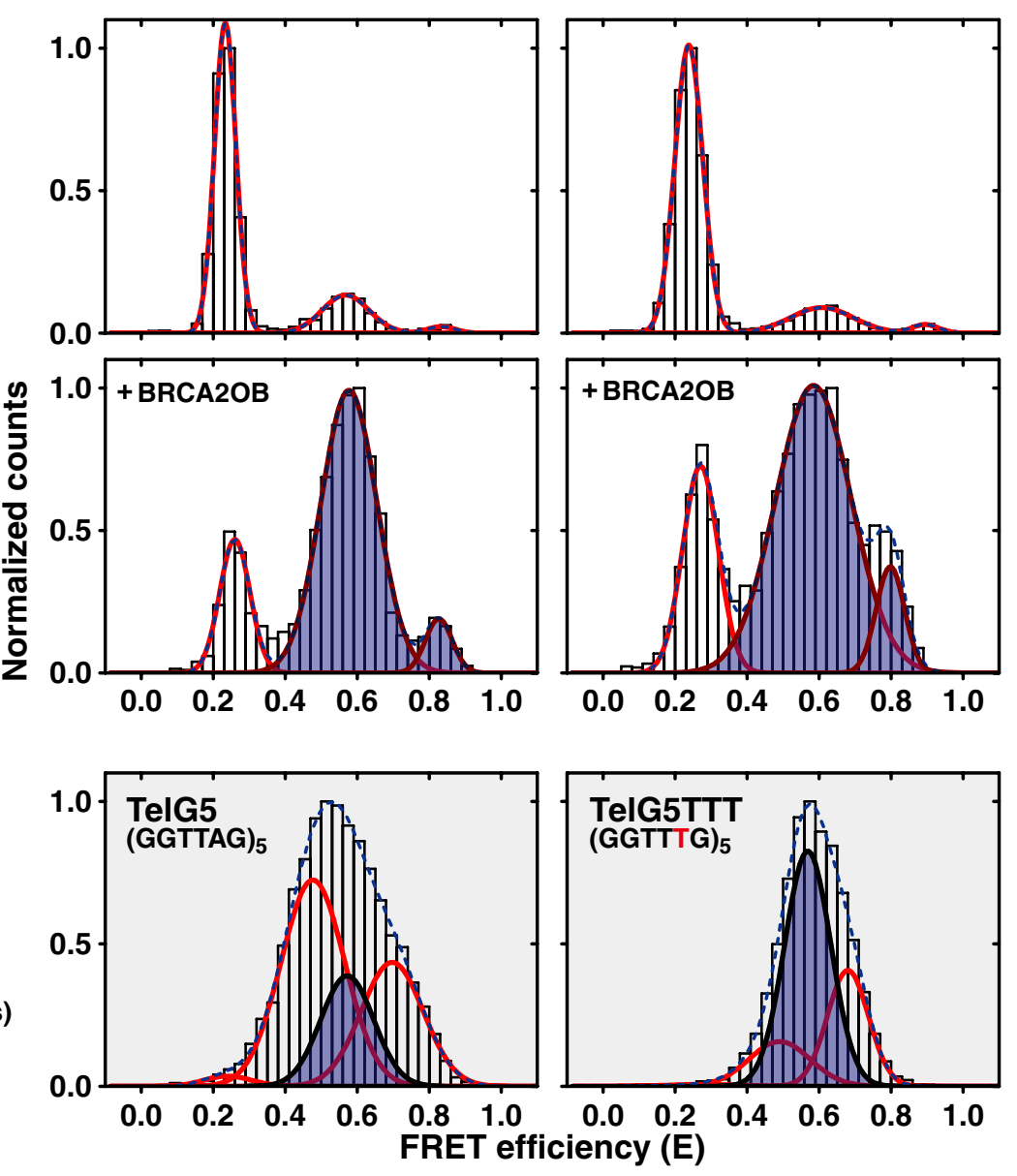

$\mathbf{f}$

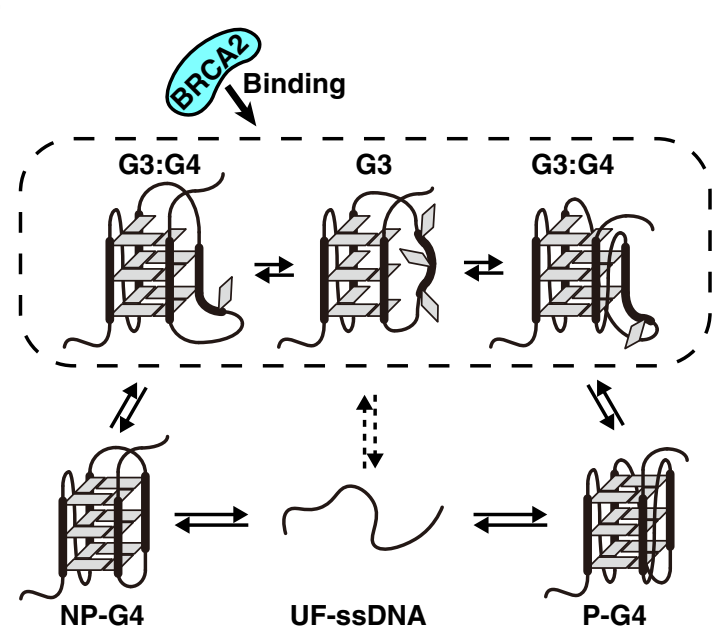


Figure 6

a

Human MRE11(708 a.a.)

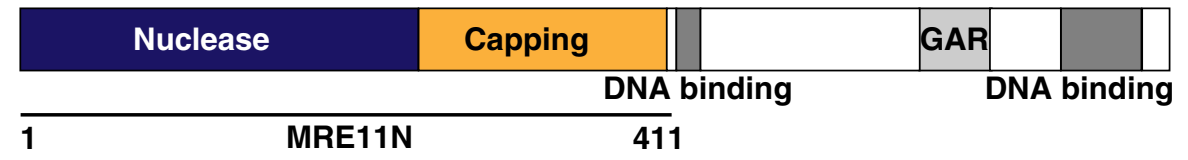

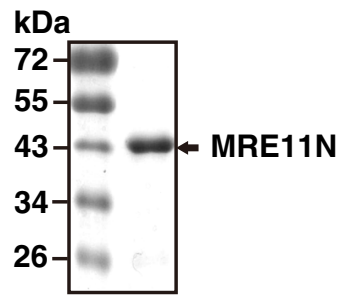

b

C TelG5-G3
$3_{3}$-( TGTTAG)-(GGTTAG)

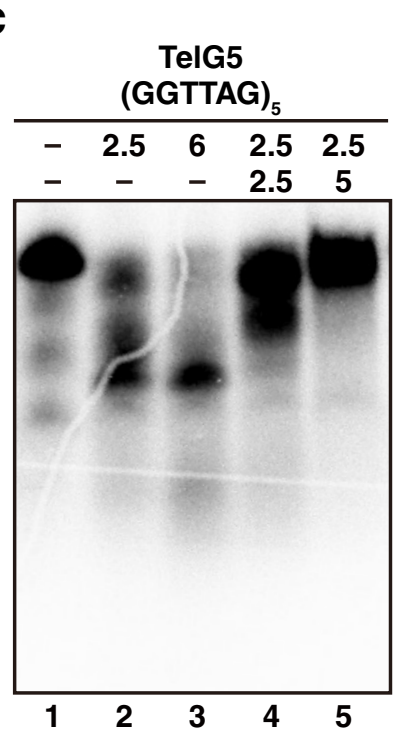

d
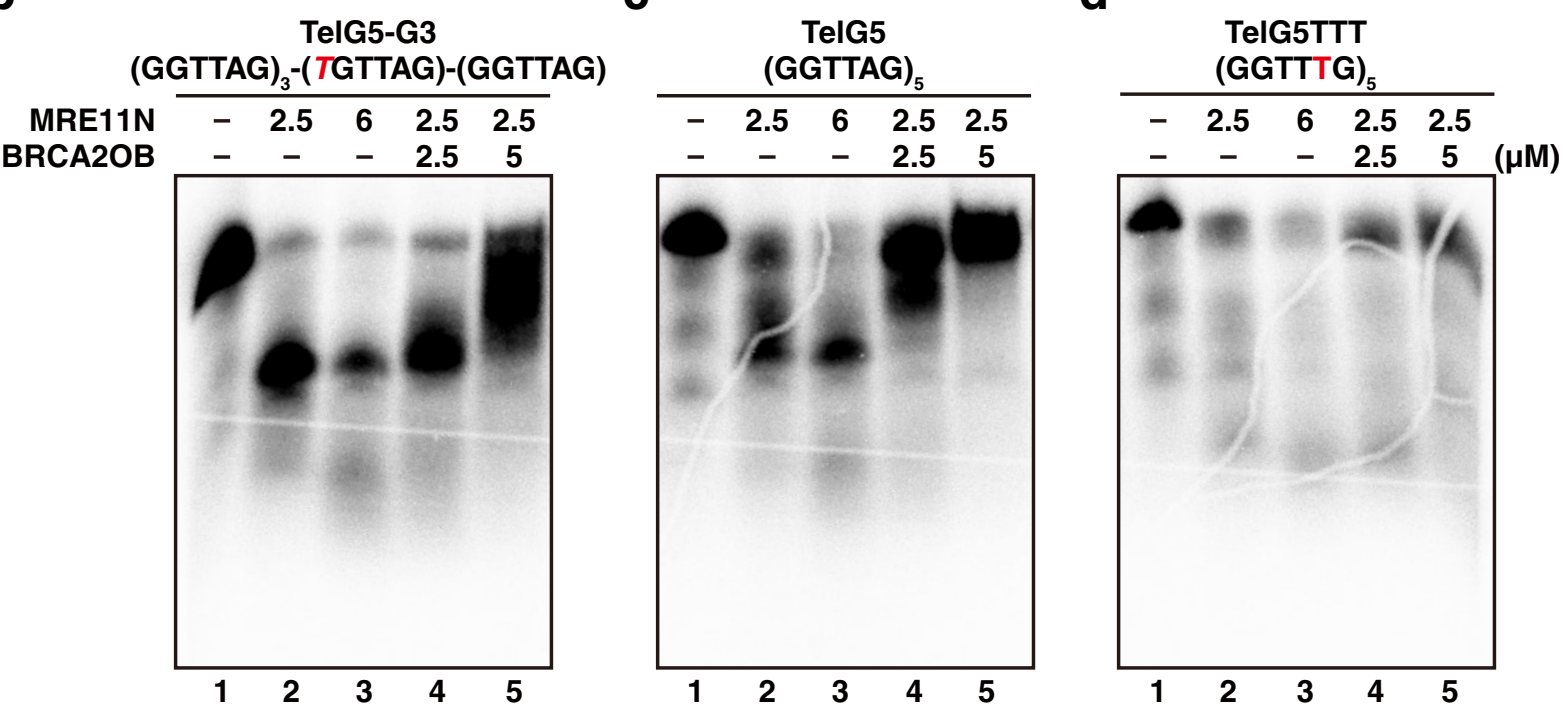

e

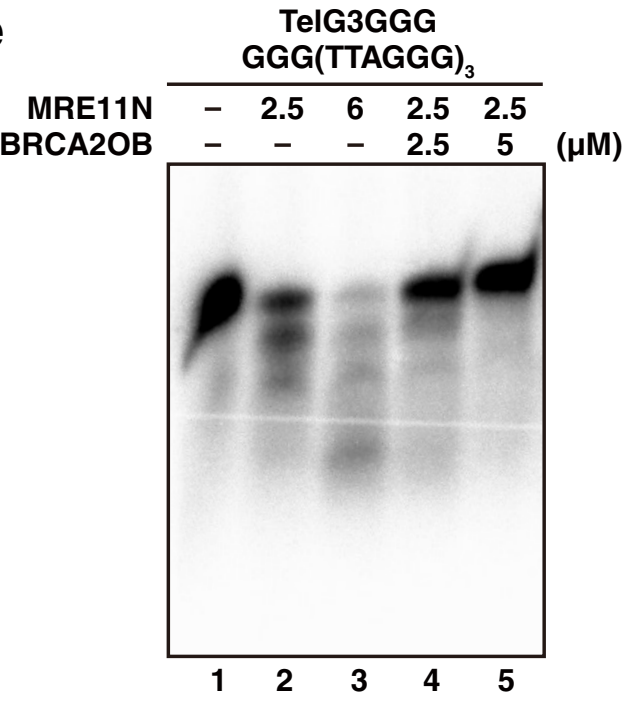


Figure 7

a

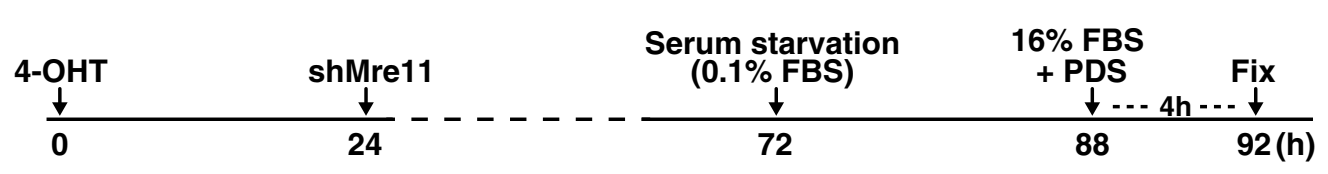

b

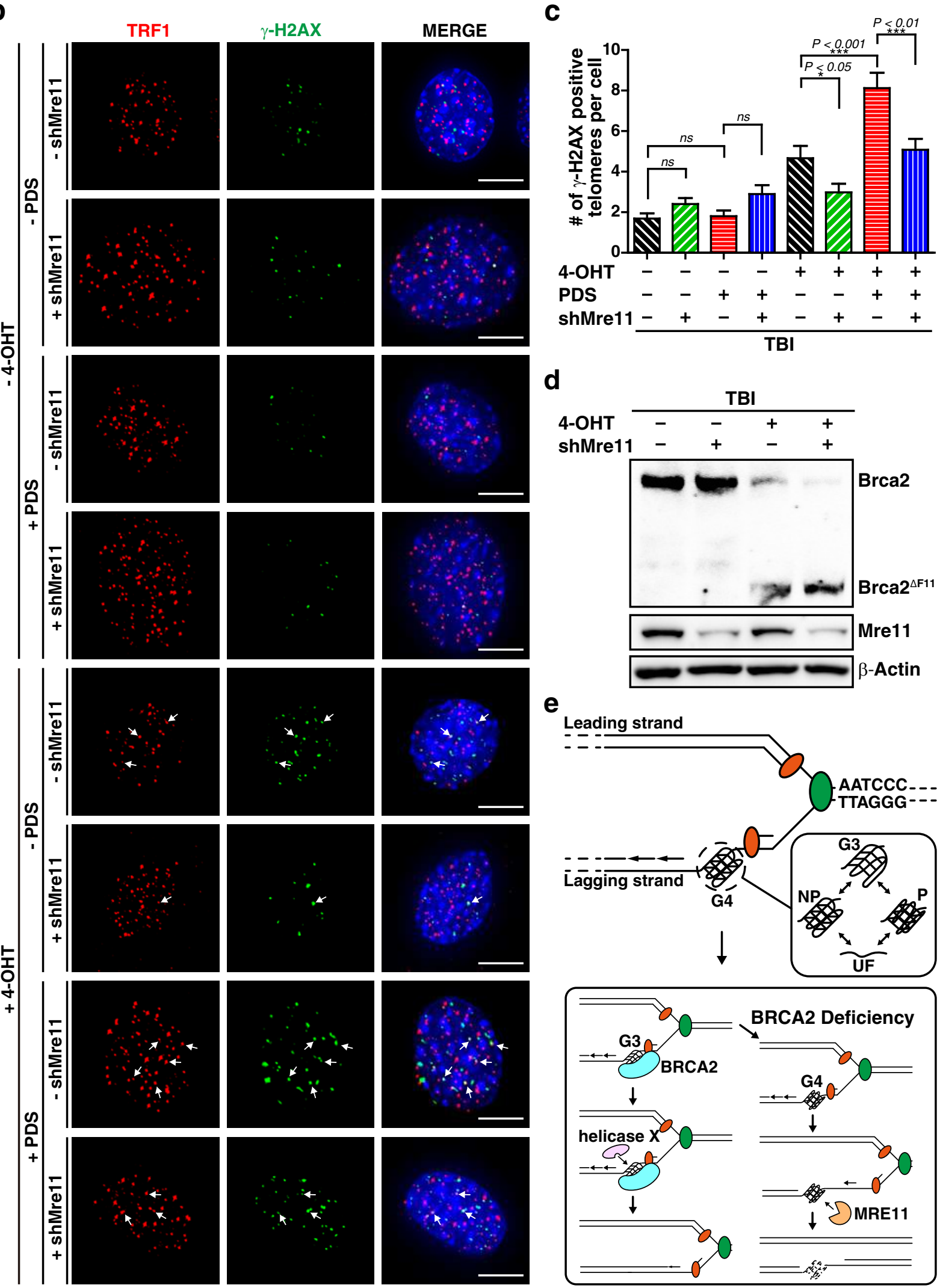


Supplementary Information for:

\title{
Dynamic interaction of BRCA2 with telomeric G-quadruplexes underlies the telomere replication homeostasis
}

\author{
Junyeop Lee ${ }^{I \S}$, Keewon Sung ${ }^{2 \S}$, So Young Joo ${ }^{1}$, Jun-Hyeon Jeong ${ }^{l}$, Seong Keun Kim ${ }^{2 *}$, and Hyunsook Lee ${ }^{l^{*}}$ \\ ${ }^{1}$ Department of Biological Sciences \& IMBG, Seoul National University, Seoul 08826, South Korea \\ ${ }^{2}$ Department of Chemistry, Seoul National University, Seoul 08826, South Korea
}

$\S$ These authors contributed equally.

*Co-correspondence: S.K.K. (ㄹeongkim@snu.ac.kr) \& H.L. (h1212@snu.ac.kr) 
a
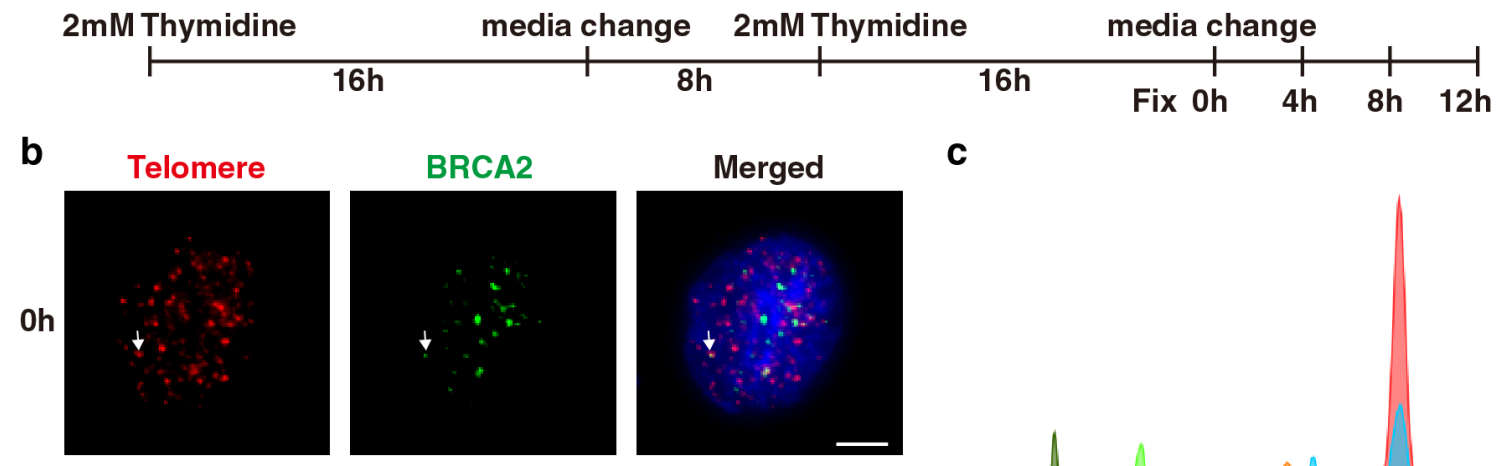

C
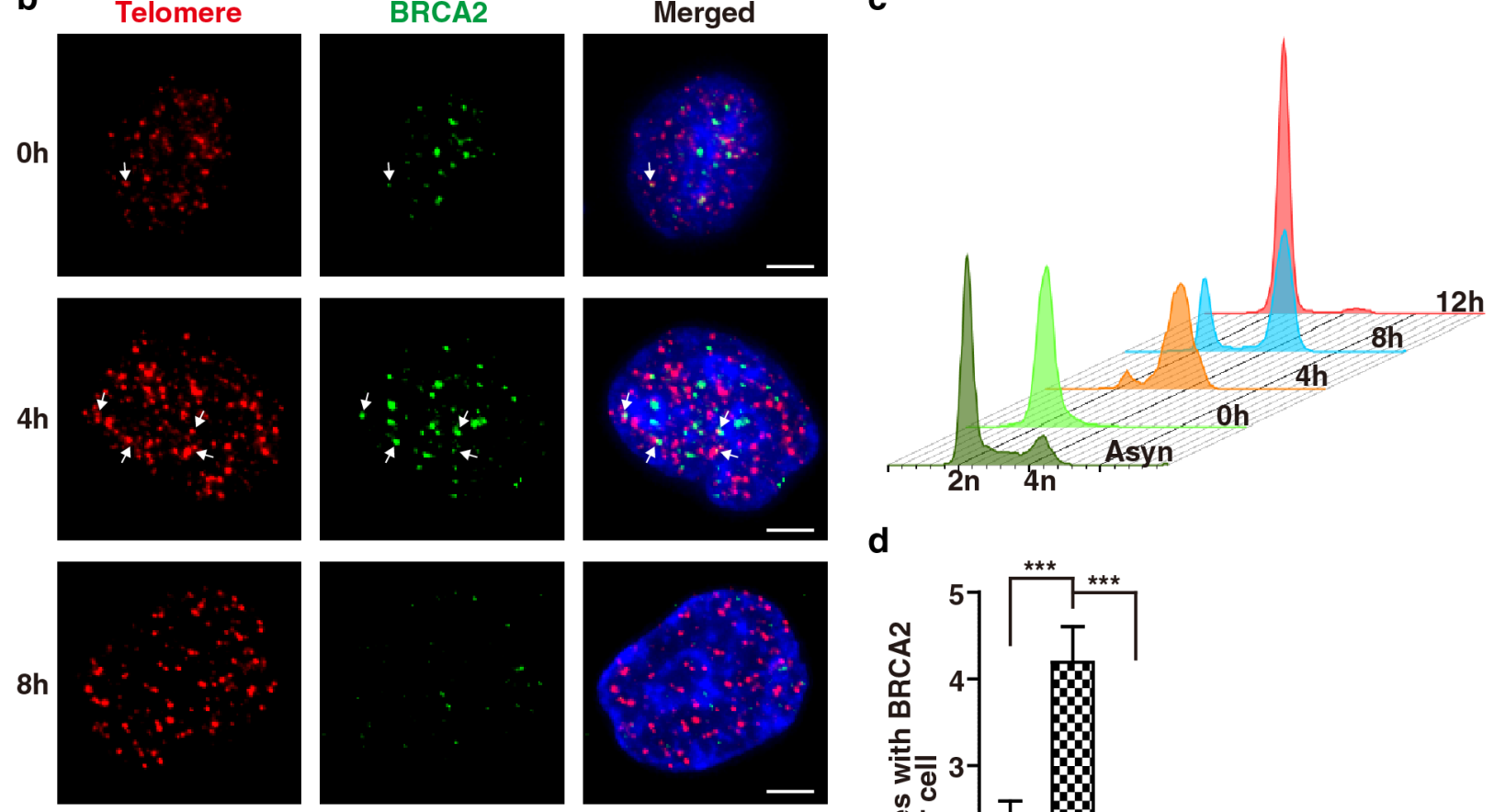

d
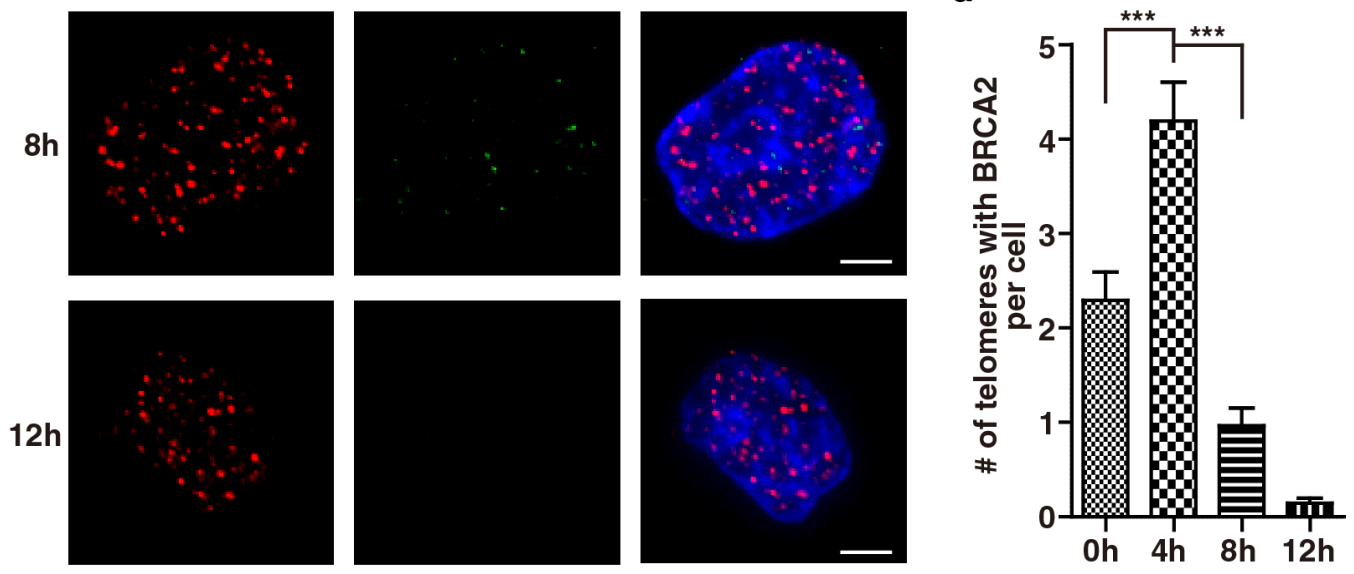

Supplementary Figure S1. BRCA2 localizes to telomeres in S-phase. (a) Schematic workflow of the experiment. NFLAP-BRCA2 HeLa cells ${ }^{1}$ were synchronized in G1/S using a thymidine double block and then washed to release into the cell cycle. Cells were then fixed at indicated time points for immunostaining or for flow cytometry. (b) Immunostaining coupled with FISH analysis. Immunostaining with anti-GFP antibody was performed to detect BRCA2, followed by denaturation and FISH analysis with a PNA-labeled telomere probe. Green, BRCA2; Red, telomere. (c) Cells were subjected to propidium iodide staining and to cell cycle analysis using flow cytometry. (d) Scoring of telomeres positive for BRCA2 after immunoFISH. Number of cells analysed: $0 \mathrm{~h}, \mathrm{n}=55 ; 4 \mathrm{~h}, \mathrm{n}=58$; $8 \mathrm{~h}, \mathrm{n}=56 ; 12 \mathrm{~h}, \mathrm{n}=62$. White scale bar, $5 \mu \mathrm{m}$. 


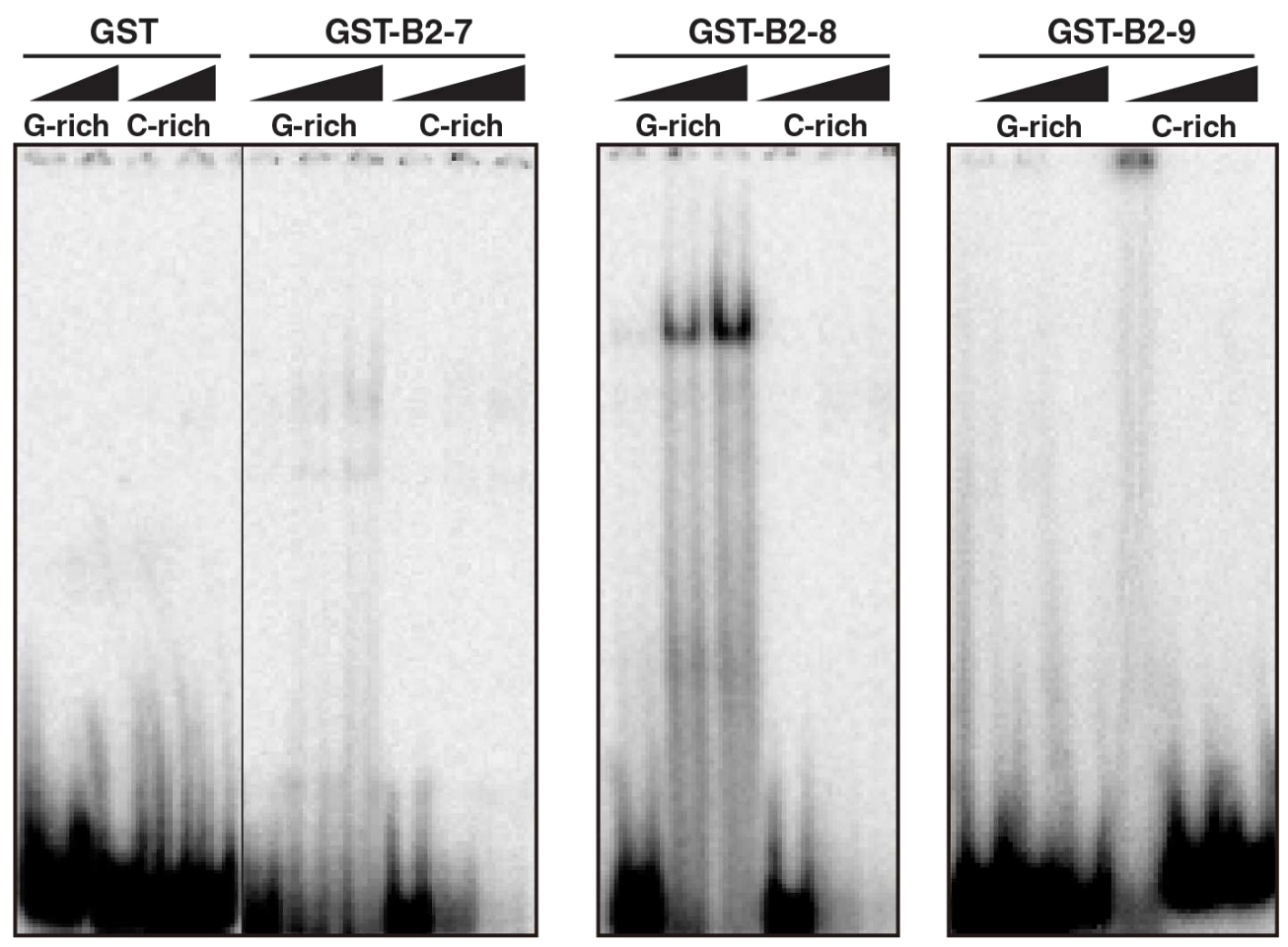

Supplementary Figure S2. The OB-fold-containing fragment of BRCA2 binds to the telomere Grich strand. GST-tagged B2-7 (hBRCA2 2438-2824), B2-8 (hBRCA2 2780-3197), and B2-9 (hBRCA2 3189-3418), which include part of the BRCA2 ssDNA binding domain (DBD), were purified from E. coli. Only the GST-B2-8, containing the OB-folds, bound to the telomeric G-rich strand (5'GCC CGC GGT TAG GGT TAG GGT TAG GGT TAG GGG TGA ATT C-3') in a concentrationdependent manner. GST-B2-8 does not bind to the C-rich complementary strand (5'-GAA TTC ACC CCT AAC CCT AAC CCT AAC CCT AAC CGC GGG C-3'). The reaction buffer contained $150 \mathrm{mM}$ $\mathrm{KCl}$. 


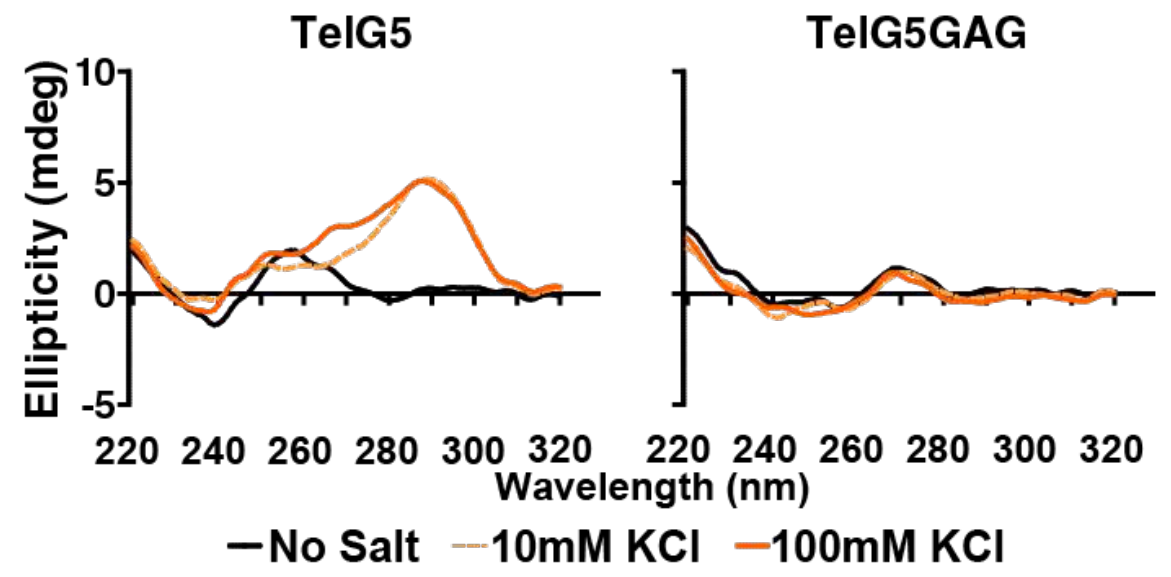

Supplementary Figure S3. Conformation of TelG5 and TelG5GAG revealed by CD spectra. CD (Circular Dichroism) spectra of TelG5 and TelG5GAG were analyzed under three conditions; no salt (black), $10 \mathrm{mM} \mathrm{KCl}$ (yellow, dashed), and $100 \mathrm{mM} \mathrm{KCl}$ (orange). In contrast to TelG5, in which the ellipticity increases substantially at higher concentration of $\mathrm{KCl}$, no significant change was observed for TelG5GAG, indicative of the unfolded structure. 


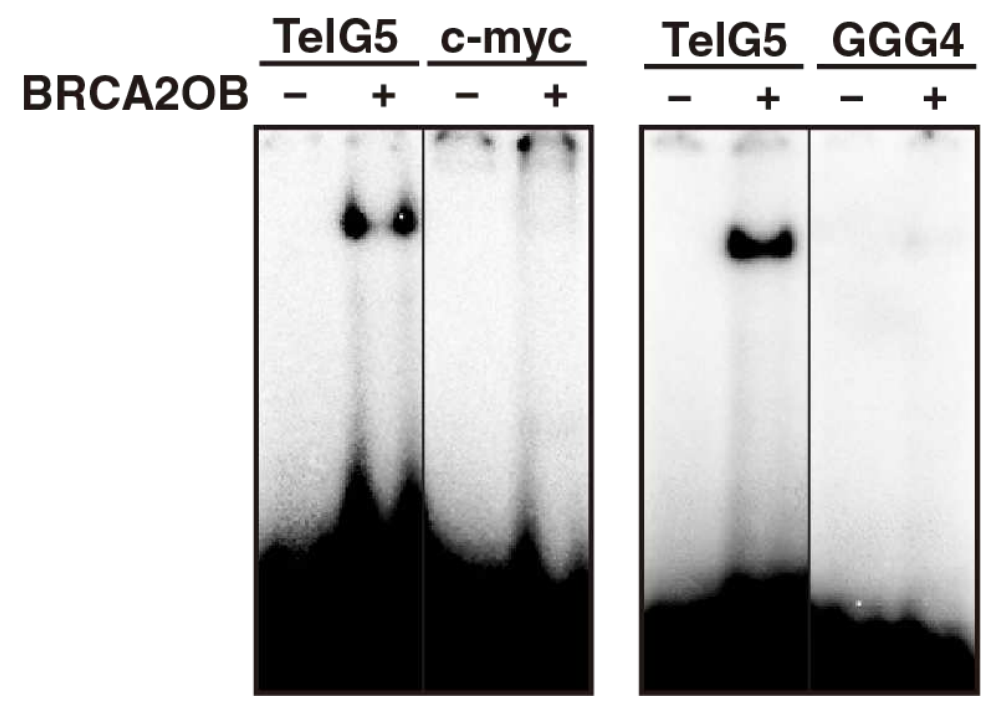

Supplementary Figure S4. BRCA2OB specifically interacts with telomeric G4. Recombinant BRCA2OB was incubated with radiolabeled TelG5, G4-forming C-MYC promoter (c-myc) or an artificial sequence designed to fold into G4, and subjected to EMSA. BRCA2OB specifically binds to TelG5. The reaction buffer contained $10 \mathrm{mM} \mathrm{Na}^{+}$but no $\mathrm{K}^{+}$ions. 


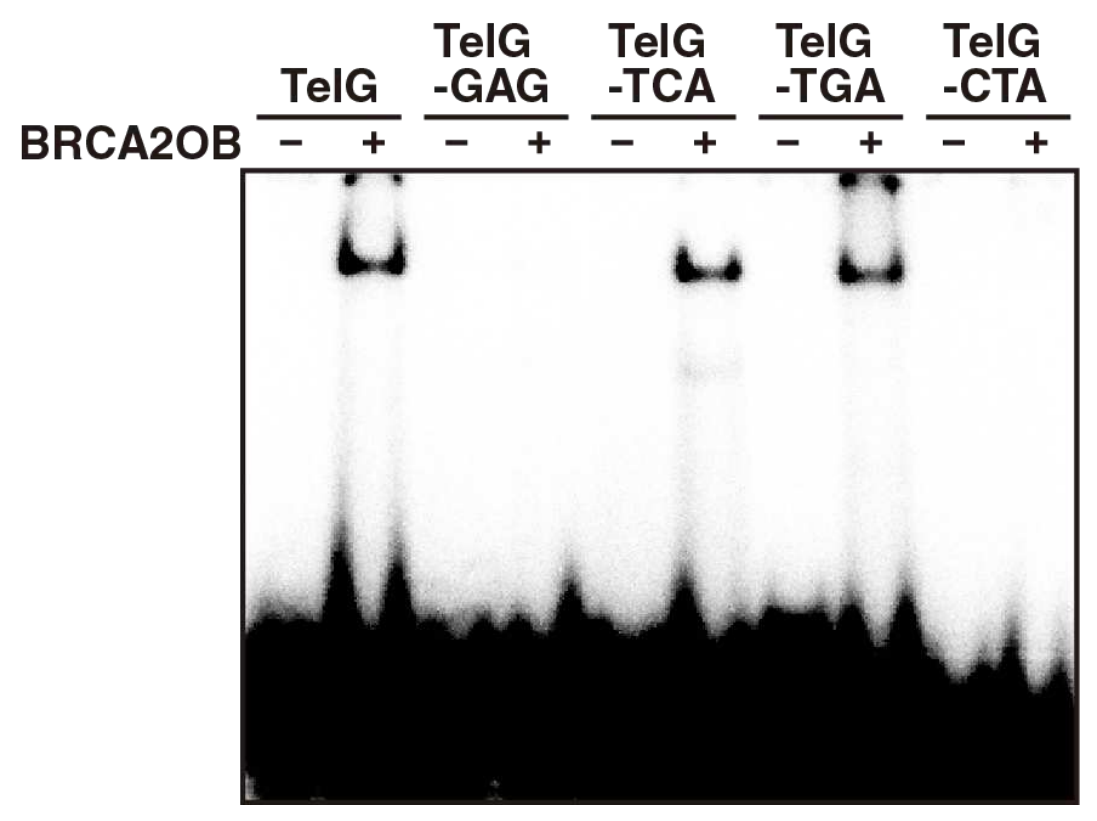

Supplementary Figure S5. Differential binding profile of BRCA2OB to telomere variants. EMSA was performed with several telomere variant repeats. TelG-TCA, -TGA, and -CTA represent telomere variants of wild-type TelG, where TTA is substituted to TCA, TGA, and CTA, respectively (Supplementary Table 1). TelG-GAG is the unfolded mutant control, as the GGG repeat is substituted with GAG. The reaction buffer contained $10 \mathrm{mM} \mathrm{Na}^{+}$. 

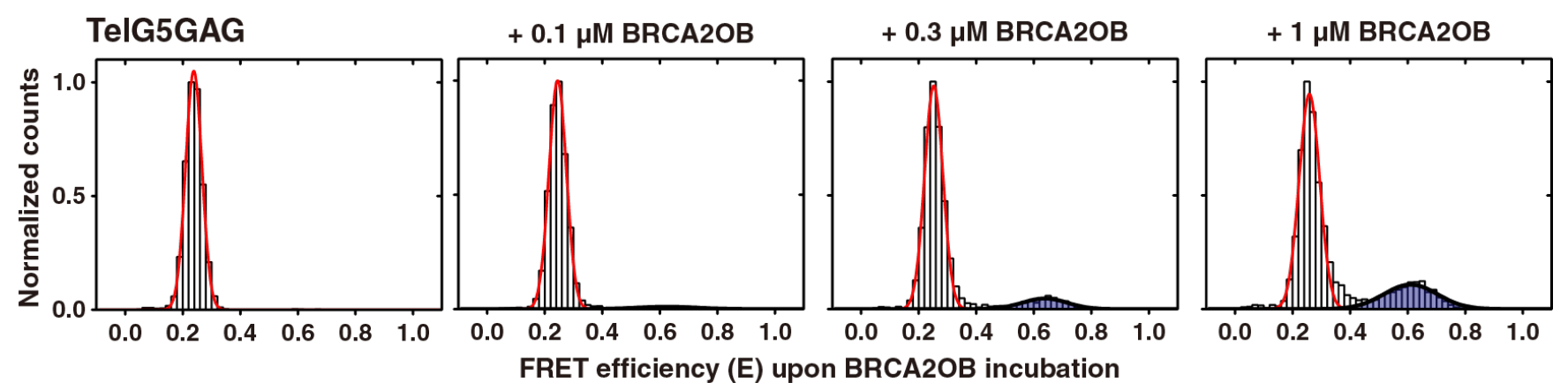

Supplementary Figure S6. Interaction of BRCA2OB with TelG5GAG. Gaussian fitted smFRET histograms of TelG5GAG with increasing concentrations of BRCA2OB (color-coded as in Fig. 3). Negligible binding $(<3 \%)$ was observed in $0.1 \mu \mathrm{M}$ BRCA2OB, and the bound fraction remained marginal ( 20\%) even after increasing BRCA2OB concentration to $1 \mu \mathrm{M}$. 


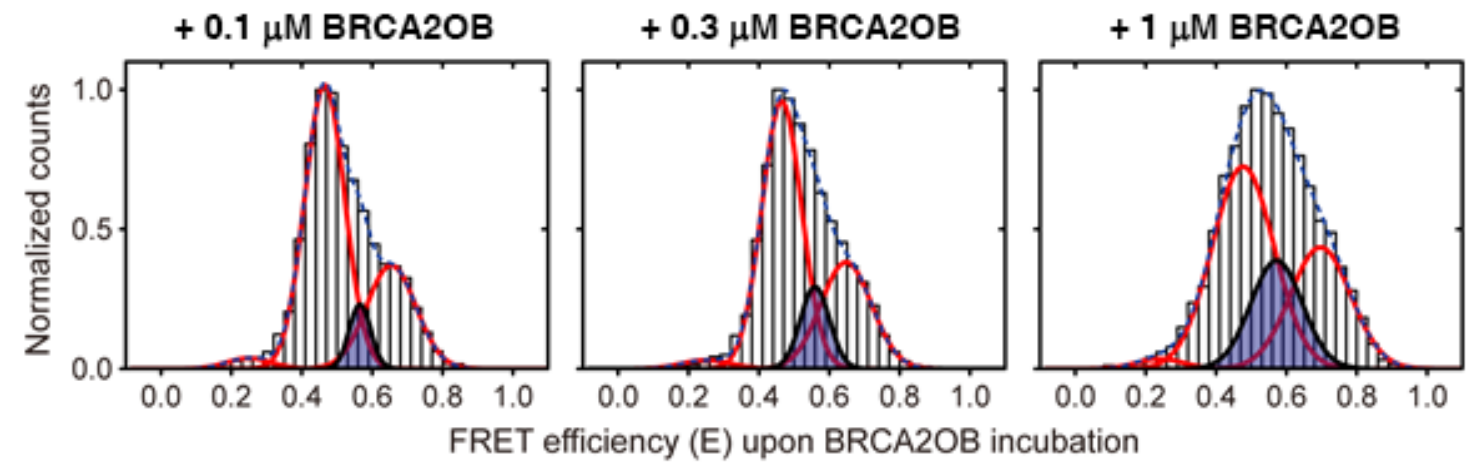

Supplementary Figure S7. BRCA2OB-bound (BD) fraction increases in a concentration-dependent manner. Gaussian fitted smFRET histograms of TelG5 upon incubation with BRCA2OB at increasing concentrations; $0.1 \mu \mathrm{M}, 0.3 \mu \mathrm{M}$, and $1 \mu \mathrm{M}$ (color-coded as in Fig. 3). 

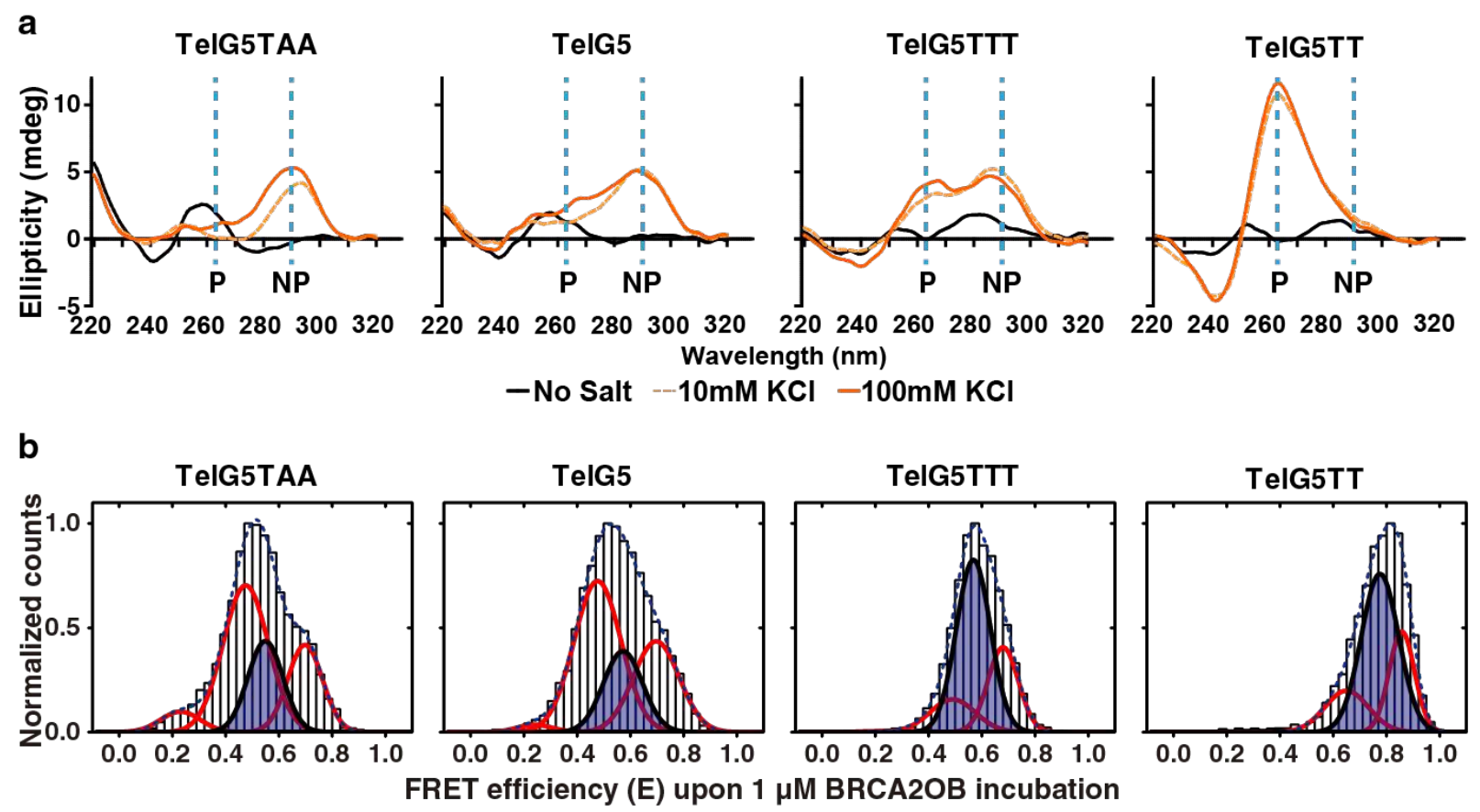

Supplementary Figure S8. Mode of BRCA2OB-binding to telomeric G4 variants. (a) CD spectra of various G4 constructs. CD spectra of four different G4-forming oligonucleotides (TelG5TAA, TelG5, TelG5TTT, and TelG5TT) in no salt (black), $10 \mathrm{mM} \mathrm{KCl} \mathrm{(yellow,} \mathrm{dashed),} \mathrm{and} 100 \mathrm{mM} \mathrm{KCl} \mathrm{(orange).}$ (b) Gaussian fitted smFRET histograms of the four G4 constructs upon incubation with $1 \mu \mathrm{M}$ BRCA2OB. Note the emergence of a new intermediate peak after BRCA2OB addition. 


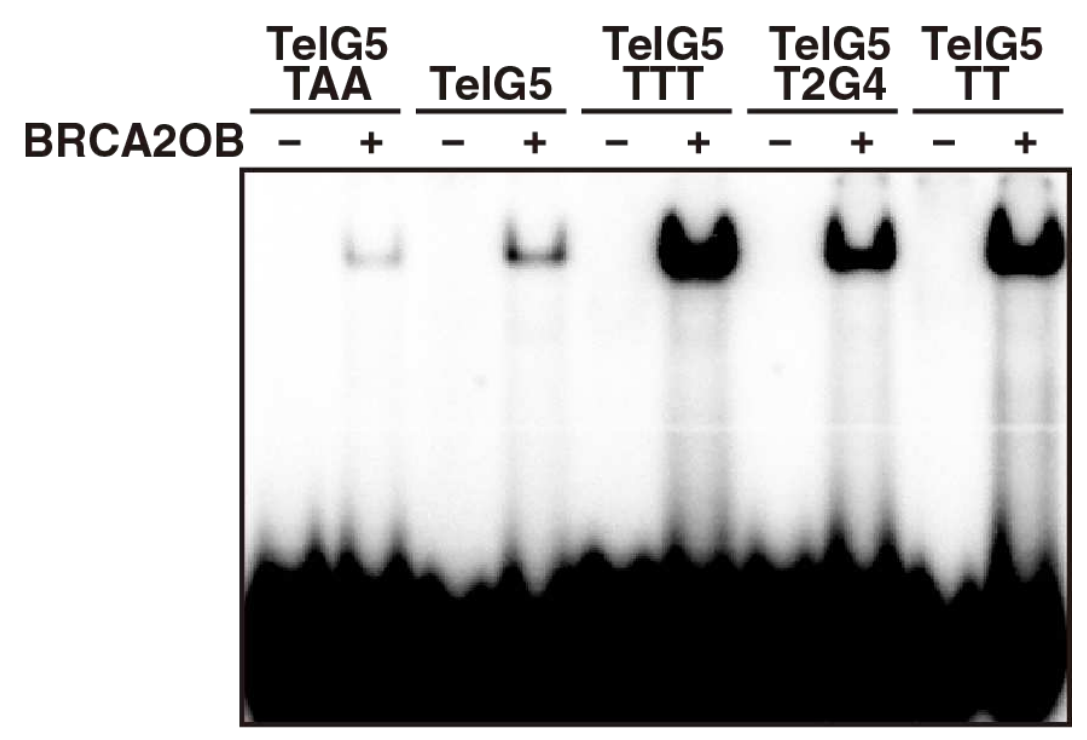

Supplementary Figure S9. Differential binding properties of BRCA2OB towards the telomeric G4 variants. EMSA of BRCA2OB with TelG5TAA, TelG5, TelG5TTT, TelG5T2G4, and TelG5TT (relative binding intensity is quantified in the bar graph in Fig. 4f and Supplementary Fig. S13d, grey bar). The reaction buffer contained $100 \mathrm{mM} \mathrm{K}$. 


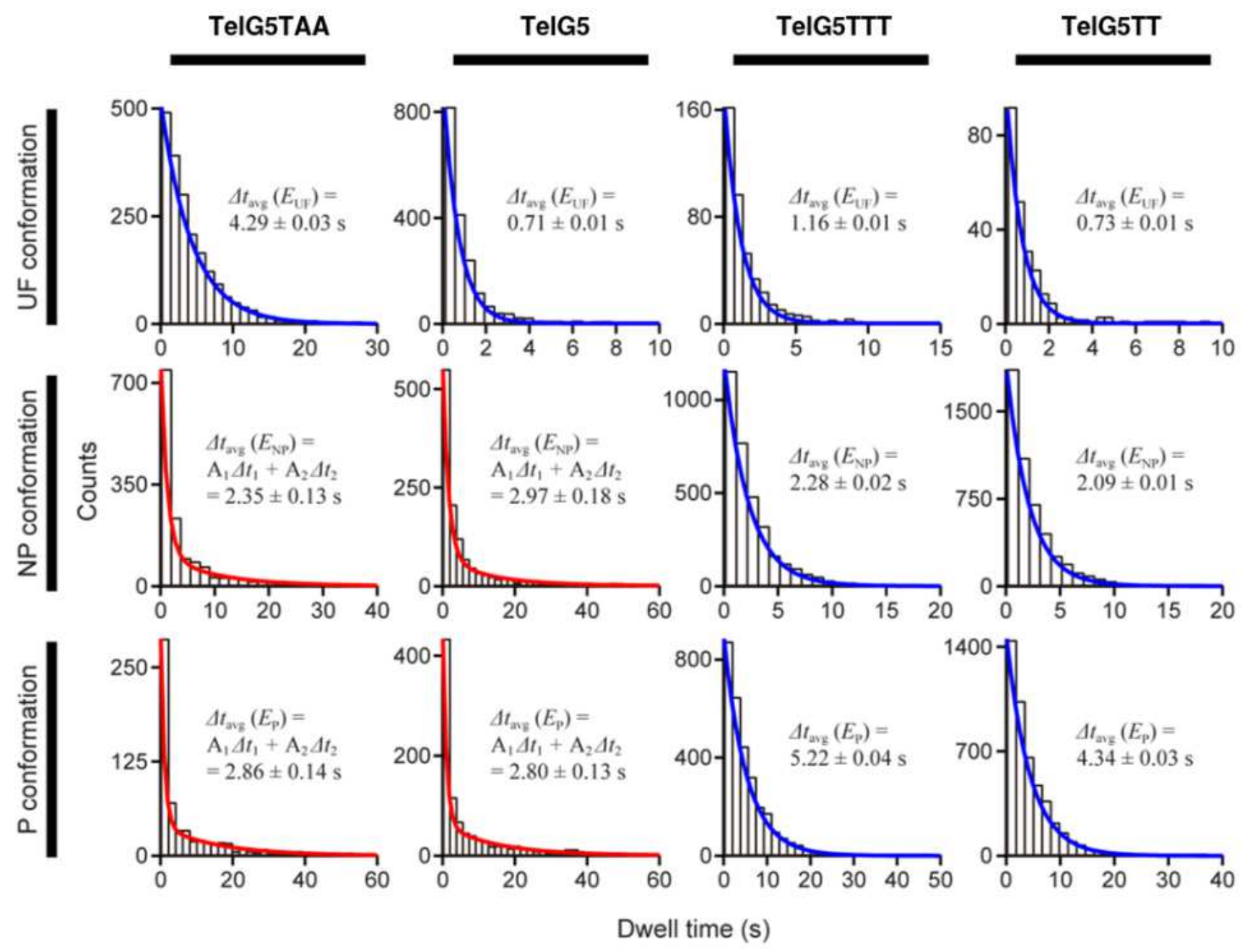

Supplementary Figure S10. Dwell time analysis of the four G4 constructs. Dwell time distributions of the four G4 constructs (TelG5TAA, TelG5, TelG5TTT, and TelG5TT) in UF (top), NP (middle), and P (bottom) conformations. The average dwell time $\left(\Delta t_{\text {avg }}\right)$ was extracted from the single-exponential fit (blue) of the UF conformation for all constructs, and NP and P conformations for TelG5TTT and TelG5TT. The dwell time distributions of NP and P conformations for TelG5TAA and TelG5 are better described using a bi-exponential fit (red), consistent with a previous report ${ }^{2}$; the average dwell time corresponds to the amplitude-weighted average value calculated by the equation at each plot. The error represents s.d. 
a

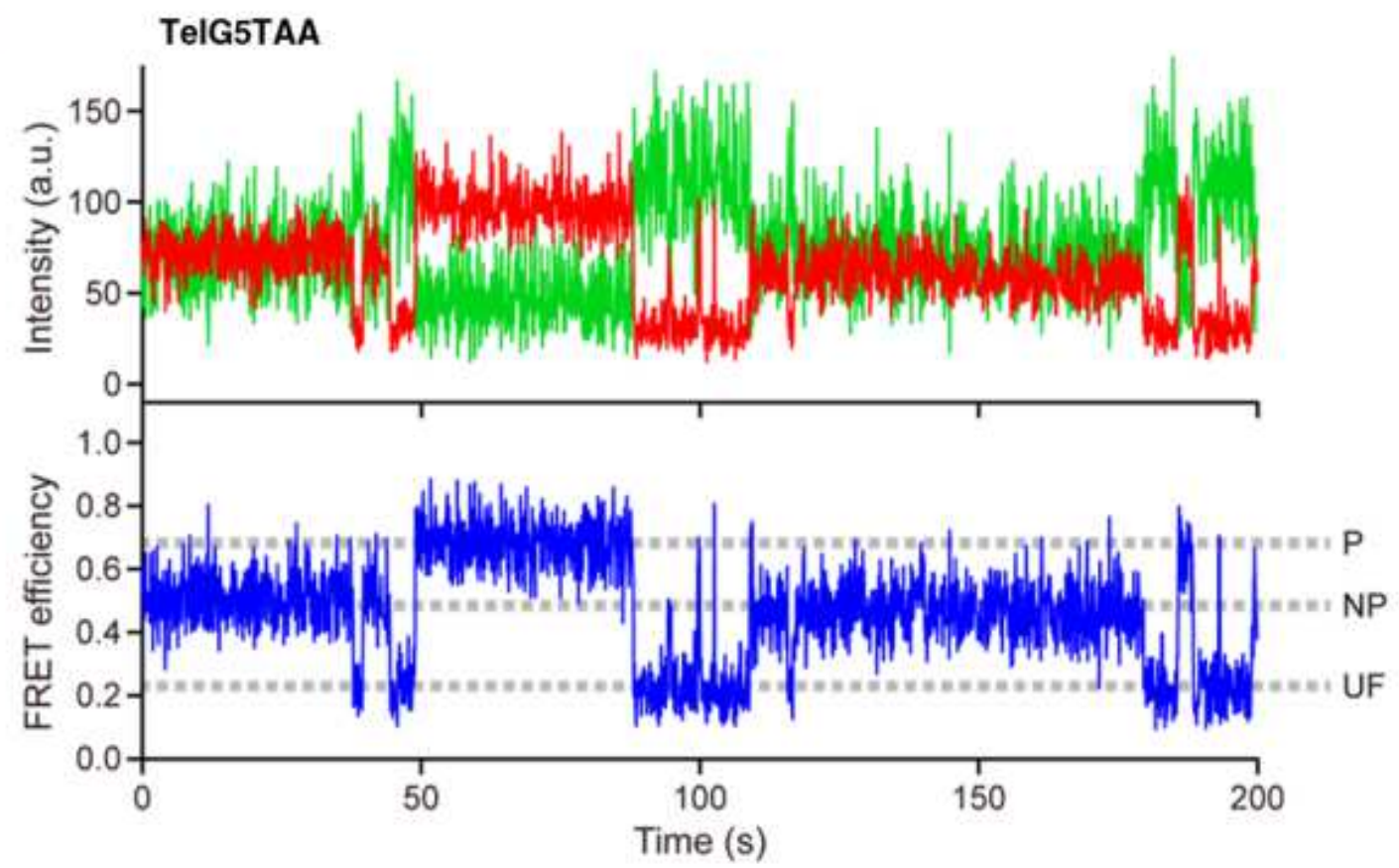

b

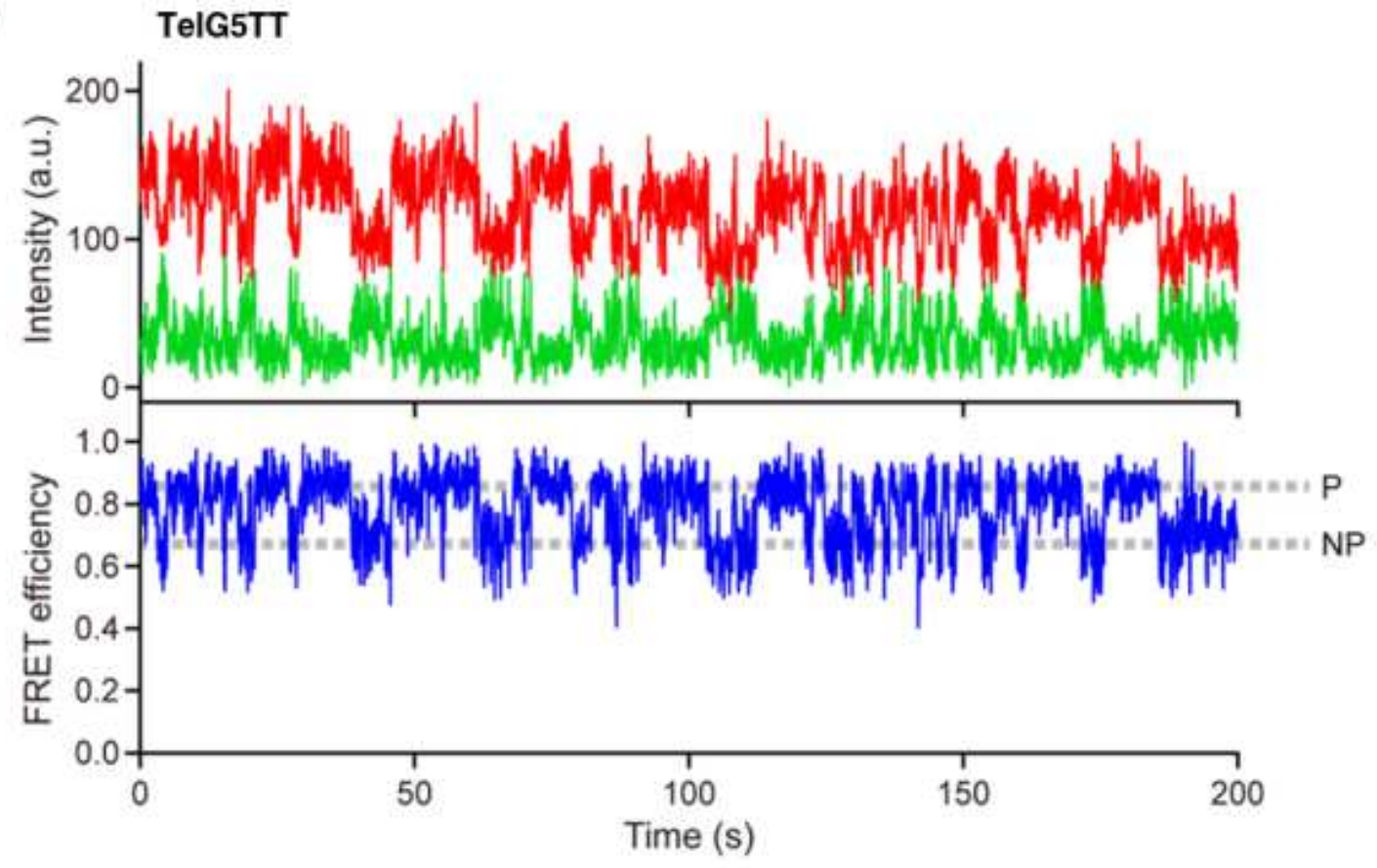

Supplementary Figure S11. Representative time trajectories of TelG5TAA and TelG5TT. (a) A representative time trajectory of TelG5TAA dynamics showing structural rearrangements that largely transition through the UF conformation. (b) A representative time trajectory of the TelG5TT molecule which transitions directly between NP and P conformations without passing through the UF conformation. 
a

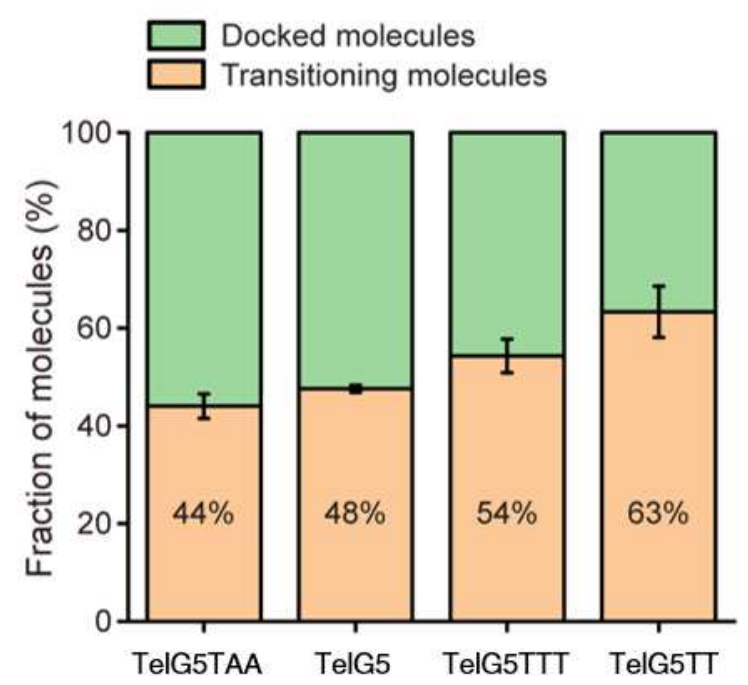

b

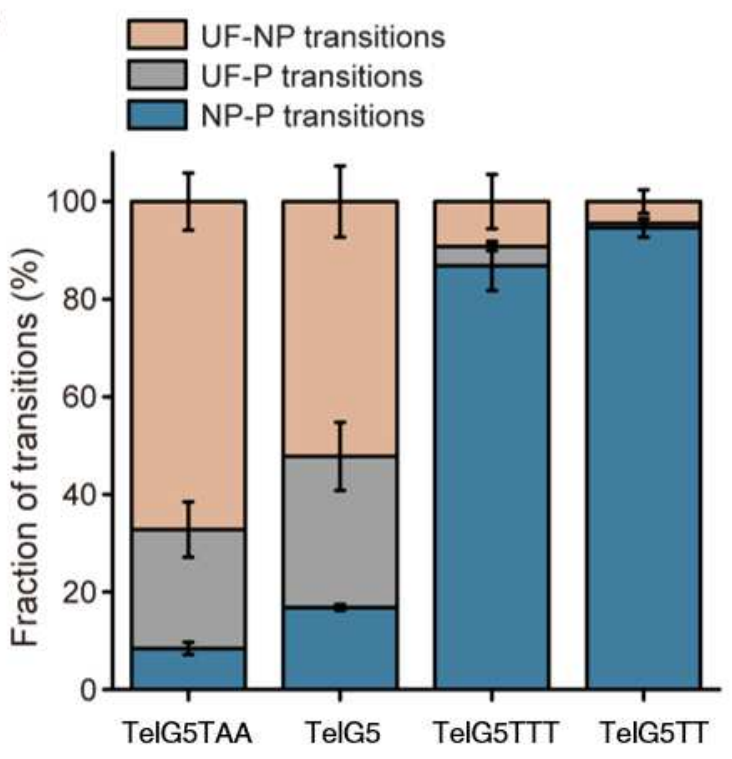

Supplementary Figure S12. Quantification of molecular transition density. (a) Fraction of G4 molecules showing time trajectories which are stably docked in a single FRET state (green) or transition between multiple FRET states (orange). (b) Relative abundance of the transitions between UF and NP conformations (UF $\rightarrow \mathrm{NP}$ and $\mathrm{NP} \rightarrow \mathrm{UF}$; beige), $\mathrm{UF}$ and $\mathrm{P}$ conformations (UF $\rightarrow \mathrm{P}$ and $\mathrm{P} \rightarrow \mathrm{UF}$; gray), and NP and $\mathrm{P}$ conformations (NP $\rightarrow \mathrm{P}$ and $\mathrm{P} \rightarrow \mathrm{NP}$; blue) quantified from the transition density plot in Fig. 4d. The density of the NP-P direct transition in Fig. $4 \mathbf{f}$ was calculated by multiplying the fraction of transitioning molecules in $\mathbf{a}$ by the fraction of NP-P transitions in $\mathbf{b}$. Error bars represent s.d. 
a

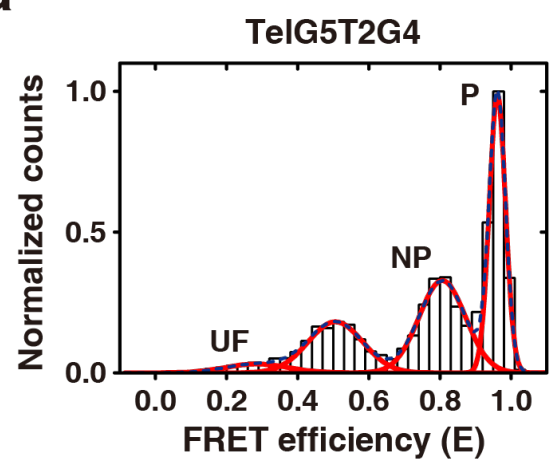

b

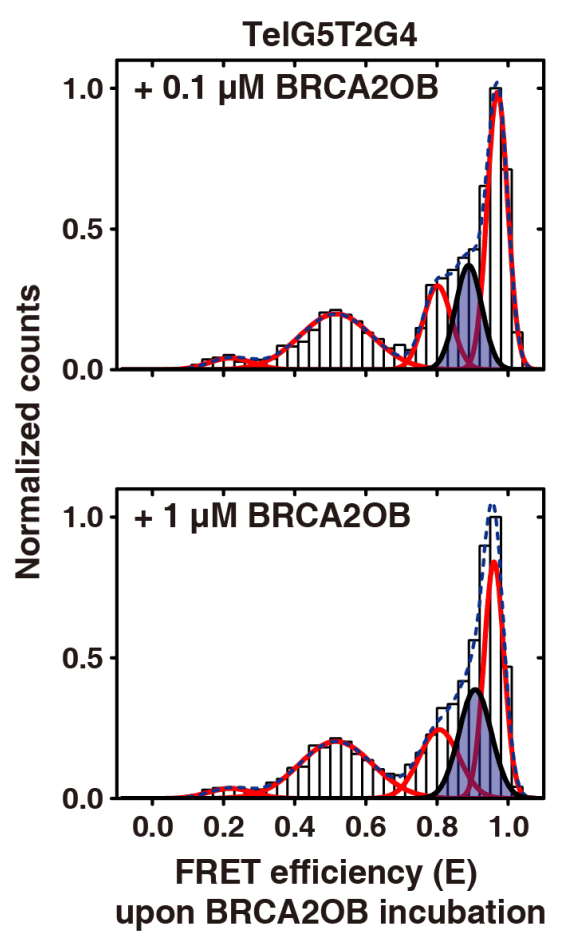

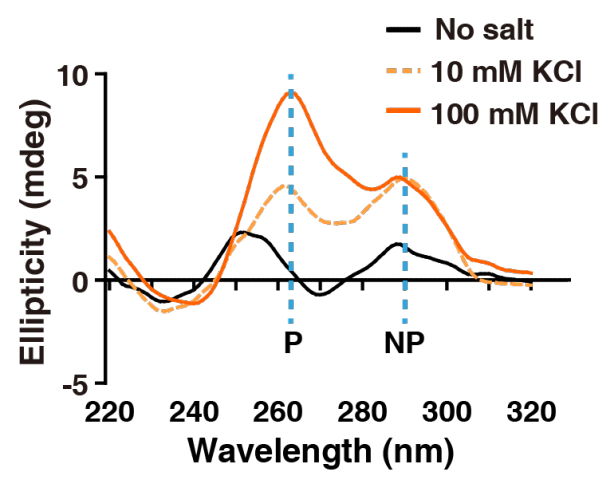

C
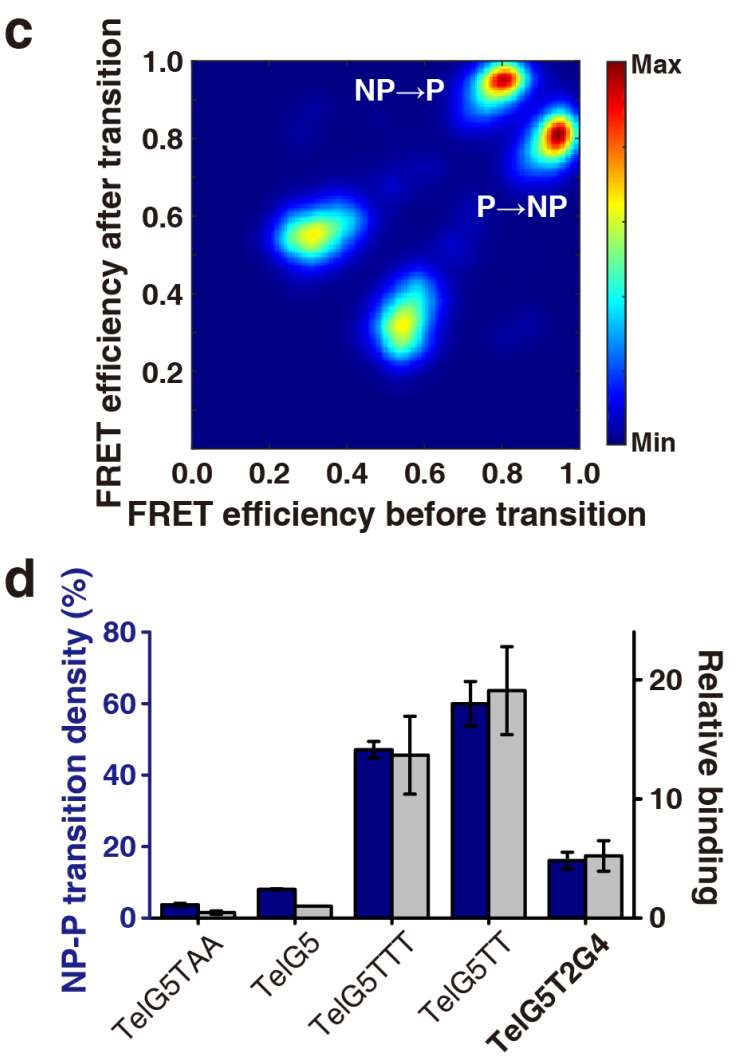

Supplementary Figure S13. Conformational dynamics and BRCA2OB-binding characteristics of TelG5T2G4. (a) A Gaussian fitted smFRET histogram of TelG5T2G4 alone (left) and its CD spectra under three conditions (right); no salt (black), $10 \mathrm{mM} \mathrm{KCl}$ (yellow, dashed), and $100 \mathrm{mM} \mathrm{KCl}$ (orange). The FRET histogram is promiscuous including an unidentified state $(E \sim 0.5)$, possibly due to the formation of four stacks of G-tetrads (i.e., the TTGGGG repeat in TelG5T2G4). (b) Gaussian fitted smFRET histograms of TelG5T2G4 upon incubation with $0.1 \mu \mathrm{M}$ (top) and $1 \mu \mathrm{M}$ (bottom) of BRCA2OB (colorcoded as in Fig. 3). (c) A transition density plot representing relative abundance of each transition among the four FRET states. (d) A plot comparing the density of direct NP-P transitions (mean \pm s.d.) calculated from the smFRET results (blue bars, left axis) and relative binding of BRCA2OB (mean \pm s.e.m.) determined from the EMSA data (gray bars, right axis). The plot is identical to Fig. $\mathbf{4 f}$, but the values of the TelG5T2G4 construct are added. 


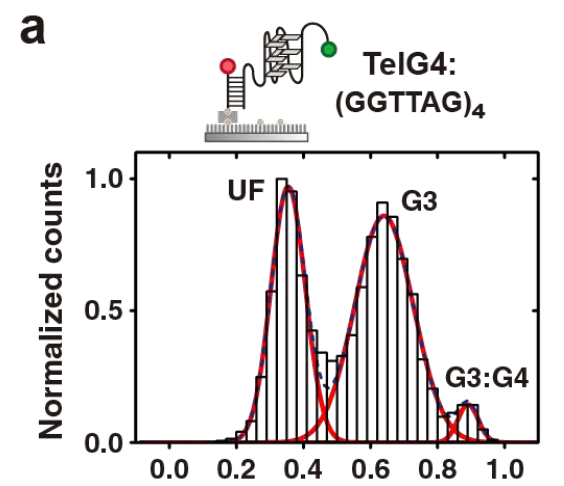

b

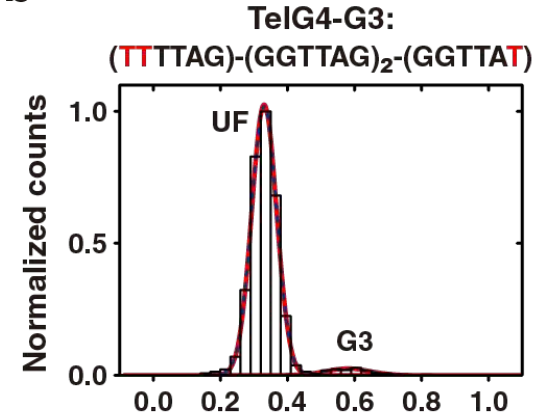

w/ $0.1 \mu \mathrm{M}$ BRCA2OB

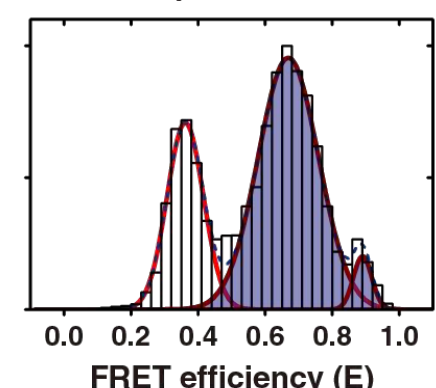

FRET efficiency (E)

w/ $0.1 \mu \mathrm{M}$ BRCA2OB

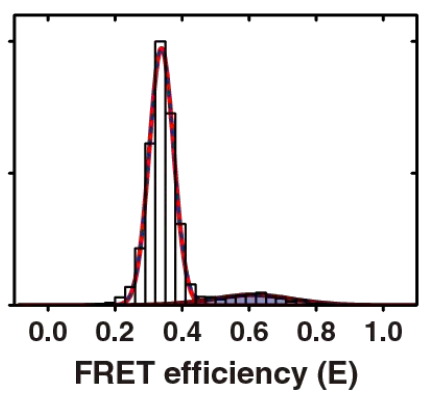

w/ $1 \mu \mathrm{M}$ BRCA2OB

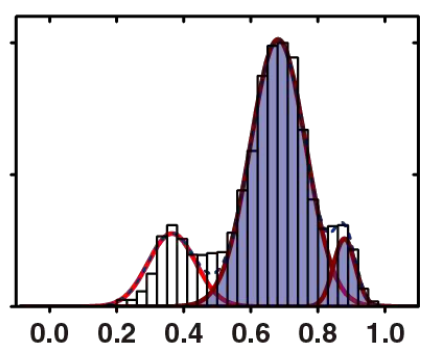

w/ 1 MM BRCA2OB

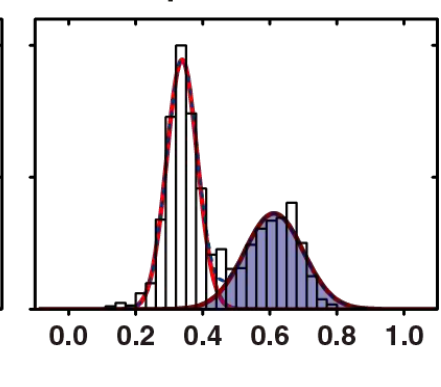

Supplementary Figure S14. Conformation and BRCA2OB binding characteristics of G3- structures.

(a) smFRET histograms of TelG4 (left) which contains three GGG triplets (cartoon at the top) similar to TelG5-G3 (Fig. 5), alone (left) and in the presence of $0.1 \mu \mathrm{M}$ (middle) and $1 \mu \mathrm{M}$ (right) of BRCA2OB. Three FRET states were observed, as seen for TelG5-G3. FRET values were slightly changed compared to TelG5-G3, due to differences in construct length. Upon addition of BRCA2OB, the middle-FRET state becomes stronger. (b) smFRET histograms of TelG4-G3, where G bases in the tail sequence other than the three GGG triplets are replaced with T, alone (left) or upon incubation with BRCA2OB at $0.1 \mu \mathrm{M}$ (middle) and $1 \mu \mathrm{M}$ (right). For TelG4-G3, a single folded state $(E \sim 0.6)$ was identified with low population $(\sim 4 \%)$, indicating that the middle-FRET state corresponds to the G3 structure. BRCA2OB effectively captured the minor population of $\mathrm{G} 3$, resulting in a bound fraction of $\sim 40 \%$ with $1 \mu \mathrm{M}$ BRCA2OB. Given that the only difference between TelG4 and TelG4-G3 is the presence of G bases in the tail sequence, the high-FRET state of TelG4 was assigned as a G3:G4 structure involving the tail that forms through interactions of G3 with extra G bases in the tails. On the basis of structural homology between TelG4 and TelG5-G3, both of which comprise three GGG triplets and the same tail sequences (i.e., GGTTA and TTAG at the 5' and 3' end, respectively), the high-FRET state of TelG5-G3 also represents the tail-associated G3:G4 conformation, while the middle-FRET state for TelG5-G3 likely consists of not only G3 but a "loop-associated" G3:G4 structure that may be assembled by the interaction between the $\mathrm{G} 3$ and remaining $\mathrm{G}$ bases in the long loop sequence (Fig. 5). 
a

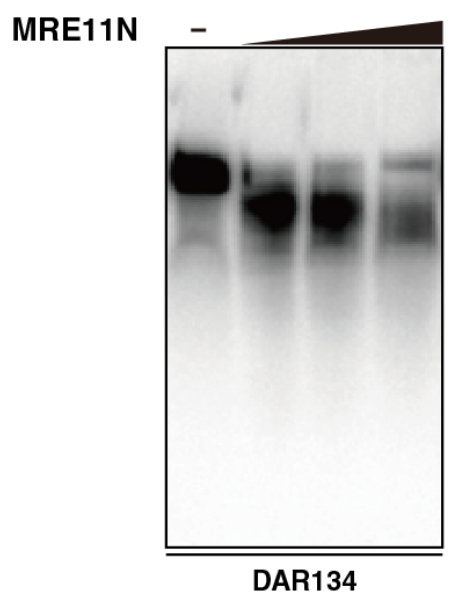

b

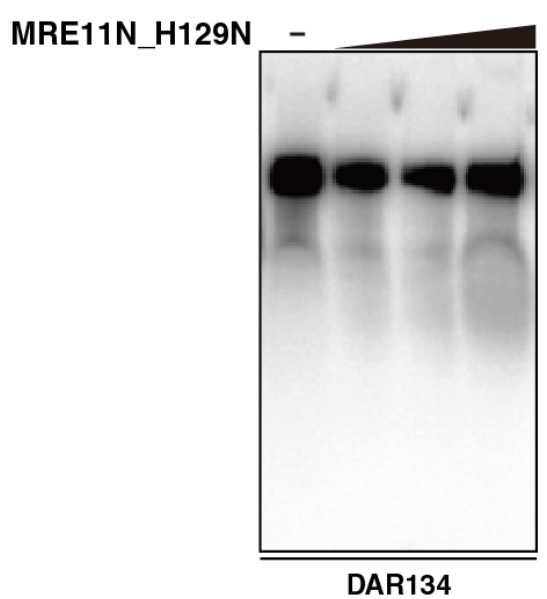

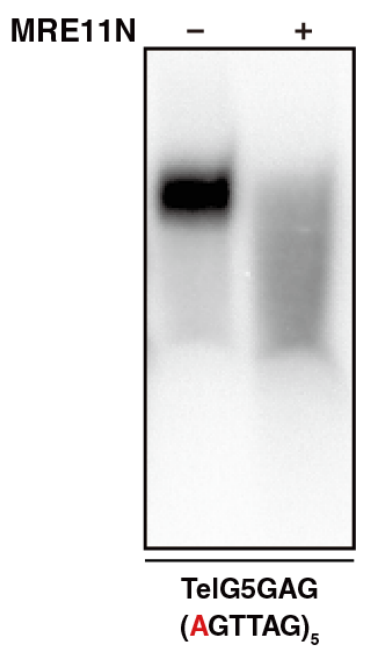

Supplementary Figure S15. Nuclease activity of the purified MRE11N. (a) Denaturing PAGE

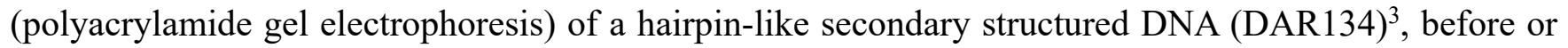
after incubation with MRE11N (left) or the nuclease-dead form Mre11N_H129N (right). Recombinant MRE11N cleaved DAR134 in a concentration-dependent manner, whereas MRE11N_H129N did not. (b) Denaturing PAGE of the effect of MRE11N on unstructured single-strand DNA (TelG5GAG). MRE11N degraded the substrate completely through its 5' to 3' exo-nuclease activity. 


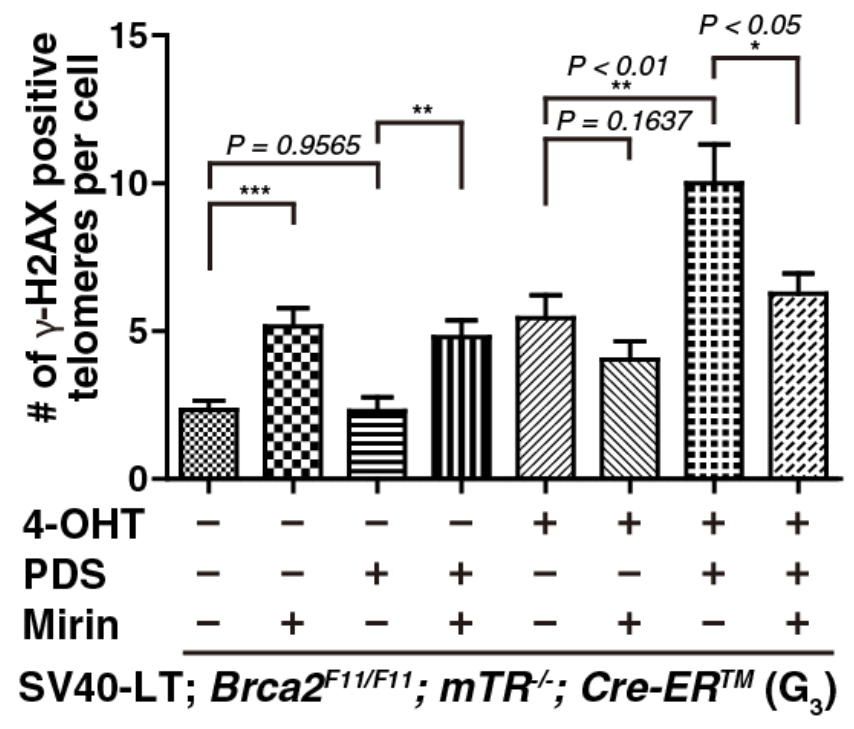

Supplementary Figure S16. Frequency of telomere damage with or without 4-OHT, PDS, or Mirin.

Mirin, the Mre11 inhibitor, showed consistent results with Mre11 depletion with lenti-shMre11 treatment (Fig. 7c). The number of cells analysed: NT (no treatment), $\mathrm{n}=46 ;+$ Mirin, $\mathrm{n}=32 ;+$ PDS, $\mathrm{n}=25 ;+$ Mirin + PDS, $\mathrm{n}=27 ;+4-\mathrm{OHT}, \mathrm{n}=32 ;+$ Mirin + 4-OHT, $\mathrm{n}=33 ;+$ PDS + 4-OHT, $\mathrm{n}=37$; + Mirin + PDS +4-OHT, $\mathrm{n}=38$. 
Supplementary Table 1. Sequences of oligonucleotides used in EMSA and nuclease assay.

\begin{tabular}{|c|c|}
\hline Name & Sequence \\
\hline TelG3 & 5' - GGT TAG GGT TAG GGT TAG - 3' \\
\hline TelG4 & 5' - GGT TAG GGT TAG GGT TAG GGT TAG - 3' \\
\hline TelG5 & 5' - GGT TAG GGT TAG GGT TAG GGT TAG GGT TAG - 3' \\
\hline TelG6 & 5' - GGT TAG GGT TAG GGT TAG GGT TAG GGT TAG GGT TAG - 3' \\
\hline TelC5 & 5' - CTA ACC CTA ACC CTA ACC CTA ACC CTA ACC - 3' \\
\hline TelG5GAG & 5' - AGT TAG AGT TAG AGT TAG AGT TAG AGT TAG - 3' \\
\hline TelG5TTT & 5' - GGT TTG GGT TTG GGT TTG GGT TTG GGT TTG - 3' \\
\hline TelG5T2G4 & 5' - GGT TGG GGT TGG GGT TGG GGT TGG GGT TGG - 3' \\
\hline TelG5TAA & 5' - GGT AAG GGT AAG GGT AAG GGT AAG GGT AAG - 3' \\
\hline TelG5TT & 5' - GGT TTG GGT TGG GTT GGG TTG GGT TTG - 3' \\
\hline TelG5-G3 & 5' - GGT TAG GGT TAG GGT TAG TGT TAG GGT TAG - 3' \\
\hline TelG3GGG & 5' - GGG TTA GGG TTA GGG TTA GGG - 3' \\
\hline c-myc & 5' - TTG AGG GTG GGG AGG GTG GGG AAT T-3' \\
\hline GGG4 & 5' - GAA CTG GGA CTG GGA TAG GGC TAG GGA AGC-3' \\
\hline TelG & 5' - GTT AGG GTT AGG GTT AGG GTT AGG GTT AG - 3' \\
\hline TelG-TCA & 5' - GTC AGG GTC AGG GTC AGG GTC AGG GTC AG - 3' \\
\hline TelG-TGA & 5' - GTG AGG GTG AGG GTG AGG GTG AGG GTG AG - 3' \\
\hline TelG-CTA & 5' - GCT AGG GCT AGG GCT AGG GCT AGG GCT AG - 3' \\
\hline TelG-GAG & 5' - GTT AGA GTT AGA GTT AGA GTT AGA GTT AG - 3' \\
\hline
\end{tabular}


Supplementary Table 2. Sequences of oligonucleotides used in single-molecule FRET assays.

\begin{tabular}{|c|c|}
\hline Name & Sequences \\
\hline TelG5 & $\begin{array}{l}\text { 5' - GCG TGG CAC CGG TAA TAG GAG ATA GGA GAG GTT AGG GTT } \\
\text { AGG GTT AGG GTT AGG GTT AG /3AmMO/ - 3, }\end{array}$ \\
\hline TelG5TAA & $\begin{array}{l}\text { 5' - GCG TGG CAC CGG TAA TAG GAG ATA GGA GAG GTA AGG GTA } \\
\text { AGG GTA AGG GTA AGG GTA AG /3AmMO/ - 3' }\end{array}$ \\
\hline TelG5TTT & $\begin{array}{l}\text { 5' - GCG TGG CAC CGG TAA TAG GAG ATA GGA GAG GTT TGG GTT } \\
\text { TGG GTT TGG GTT TGG GTT TG /3AmMO/ - 3' }\end{array}$ \\
\hline TelG5TT & $\begin{array}{l}\text { 5' - GCG TGG CAC CGG TAA TAG GAG ATA GGA GAG GTT TGG GTT } \\
\text { GGG TTG GGT TGG GTT TG /3AmMO/ - 3' }\end{array}$ \\
\hline TelG5GAG & $\begin{array}{l}\text { 5' - GCG TGG CAC CGG TAA TAG GAG ATA GGA GAA GTT AGA GTT } \\
\text { AGA GTT AGA GTT AGA GTT AG /3AmMO/ }-3 \text { ' }\end{array}$ \\
\hline TelG5T2G4 & $\begin{array}{l}\text { 5' - GCG TGG CAC CGG TAA TAG GAG ATA GGA GAG GTT GGG GTT } \\
\text { GGG GTT GGG GTT GGG GTT GG /3AmMO/ - 3' }\end{array}$ \\
\hline TelG5-G3 & $\begin{array}{l}\text { 5' - GCG TGG CAC CGG TAA TAG GAG ATA GGA GAG GTT AGG GTT } \\
\text { AGG GTT AGT GTT AGG GTT AG /3AmMO/ - 3' }\end{array}$ \\
\hline TelG5TTT-G3 & $\begin{array}{l}\text { 5' - GCG TGG CAC CGG TAA TAG GAG ATA GGA GAG GTT TGG GTT } \\
\text { TGG GTT TGT GTT TGG GTT TG } / \mathbf{3 A m M O / - 3} \text { ' }\end{array}$ \\
\hline TelG4 & $\begin{array}{l}\text { 5' - GCG TGG CAC CGG TAA TAG GAG ATA GGA GAG GTT AGG GTT } \\
\text { AGG GTT AGG GTT AG /3AmMO/ - 3' }\end{array}$ \\
\hline TelG4-G3 & $\begin{array}{l}\text { 5' - GCG TGG CAC CGG TAA TAG GAG ATA GGA GAT TTT AGG GTT AGG } \\
\text { GTT AGG GTT AT /3AmMO/ - 3' }\end{array}$ \\
\hline stem & $5^{\prime}$ - /5AmMC6/ TCT CCT ATC TCC TAT TAC CGG TGC CAC GC /Biotin/ - 3' \\
\hline
\end{tabular}

"3AmMO" and "5AmMC6" refer to amino-modification at the 3' and 5' end, respectively, for dye labeling. 


\section{References}

1. Choi, E. et al. BRCA2 Fine-Tunes the Spindle Assembly Checkpoint through Reinforcement of BubR1 Acetylation. Dev Cel/ 22, 295-308 (2012).

2. Lee, J.Y., Okumus, B., Kim, D.S. \& Ha, T. Extreme conformational diversity in human telomeric DNA. Proc Natl Acad Sci U S A 102, 18938-43 (2005).

3. Park, Y.B., Chae, J., Kim, Y.C. \& Cho, Y. Crystal structure of human Mre11: understanding tumorigenic mutations. Structure 19, 1591-602 (2011). 